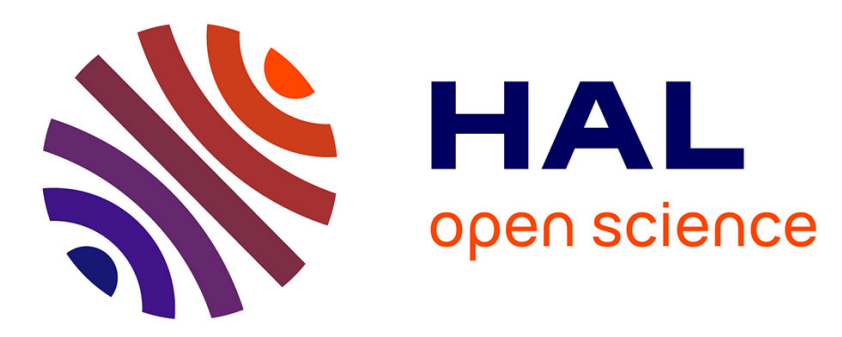

\title{
Modeling of mechanical behavior of amorphous solids undergoing fatigue loadings, with application to polymers
}

\author{
Sami Holopainen, Thierry Barriere
}

\section{- To cite this version:}

Sami Holopainen, Thierry Barriere. Modeling of mechanical behavior of amorphous solids undergoing fatigue loadings, with application to polymers. Computers \& Structures, 2018, 199, pp.57 - 73 . hal02130083

\section{HAL Id: hal-02130083 \\ https://hal.science/hal-02130083}

Submitted on 15 May 2019

HAL is a multi-disciplinary open access archive for the deposit and dissemination of scientific research documents, whether they are published or not. The documents may come from teaching and research institutions in France or abroad, or from public or private research centers.
L'archive ouverte pluridisciplinaire HAL, est destinée au dépôt et à la diffusion de documents scientifiques de niveau recherche, publiés ou non, émanant des établissements d'enseignement et de recherche français ou étrangers, des laboratoires publics ou privés. 


\title{
Modeling of mechanical behavior of amorphous solids undergoing fatigue loadings, with application to polymers
}

\author{
Sami Holopainen ${ }^{\dagger *}$, Thierry Barriere ${ }^{b *}$ \\ ${ }^{\dagger}$ Tampere University of Technology \\ Department of Civil Engineering \\ P.O. Box 600, FI-33101 Tampere, Finland \\ ${ }^{b}$ FEMTO-ST Institute, Applied Mechanics Department \\ 24 Rue de lEpitaphe, 25000 Besanon, France
}

\begin{abstract}
An approach suitable for modeling viscoelastic-viscoplastic response with isothermal fatigue damage in amorphous solids is proposed. The theory explicitly accounts for frame-indifference and dependence of the free energy on both the viscoelastic-viscoplastic deformation and fatigue damage in a thermodynamically consistent manner. The damage evolution per se is formulated by utilizing an endurance surface that shifts in an effective stress space independent on damage. The idea is suitable for solids in which the fatigue behavior is ductile, i.e. localized damage during the creation of micro-cracks governs majority (up to $95 \%$ ) of the total fatigue life. Based on implicit numerical integration, the solution procedure is presented, and the capability for technologically important polycarbonate (PC) polymer is addressed. To simulate the fatigue in real specimens, the approach is implemented in a finite-element program. The results show that fatigue life can be predicted using a single point at which fatigue most intensively initiates. A microscopic, rectangular region representing a RVE of the specimen is also investigated. Simulations, in accordance with the model assumption, indicate that damage develops in small zones around involved inhomogeneities while majority of the material remains undamaged.
\end{abstract}

Keywords: low- to high-cycle fatigue, viscoelastic-viscoplastic, thermodynamics, amorphous, simulation testing

\section{Introduction}

Fatigue denotes the cyclic deformation behavior of materials that may significantly reduce the service life of engineering components at stress levels well

*Corresponding author, e-mail: sami.holopainen@tut.fi

Preprint submitted to Computers \& Structures

December 8, 2017 
below the nominal yield strength. Economical, energy efficient, and recyclable design under such conditions favors components made of materials that enable reaching the targeted strength and elongation properties in an optimal manner. Manufacturers are interested in the fatigue resistance, especially when the service life is difficult to inspect or fatigue may cause an accident under service, Maxwell et al. (2005). In fact, for a long time, the service failures of engineering components owing to mechanical fatigue have been estimated to be the most dominant cause of remarkable financial losses, Ritchie (1999); Beesley et al. (2017). Moreover, time-consuming and costly testing of long-term fatigue life exposed to environmental conditions and previous operation histories can capitalize capable models and the high computational power currently procurable.

Examples of materials having an excellent combination of strength and toughness are amorphous polymers and amorphous metals or alloys, termed bulk metallic glasses (BMGs). However, amorphous solids, particularly BMGs, are susceptible to cyclic fatigue damage that emerges as a low endurance limit, e.g. Launeya et al. (2009); Sun and Wang (2015). Despite this feature and the motivation above, fatigue failure of amorphous solids has received little attention so far. Instead, many of the recent, appealing approaches have focused on micromechanically based damage evolution under multiaxial static loadings that may cause large deformations but not fatigue failure, Lugo et al. (2014); Wang et al. (2016); Engqvist et al. (2016). Much of recent studies has also been dedicated to the research of crack development under arbitrary fatigue loadings, see Sun and Wang (2015); Dündar and Ayhan (2015); Kanters et al. (2016); Hughes et al. (2017); Ding et al. (2017). However, referred fracture mechanics based approaches are not aimed at the crack initiation phase that can encompass the majority (even 95\%) of the entire fatigue life, Marissen et al. (2001); Janssen et al. (2008). Furthermore, research including multiaxial fatigue data for amorphous solids is nonexistent in literature.

Fatigue failure of amorphous solids is owing to either thermally or mechanically governed mechanisms, see Janssen et al. (2008); Murakami (2012). This paper is focused on the mechanically dominated fatigue that does not influence a marked temperature rise and comes forth at relatively low stress frequencies below the yield stress. Fatigue development under such conditions can be divided into two phases. During the first, initiation phase, failure is typically attributed to impurities or deficiencies producing notable stress concentrations, which may exceed the strength limits of the material, as has been noted for polymers Marissen et al. (2001); Kanters et al. (2016); Holopainen et al. (2017) and BMGs Launeya et al. (2009); Sun and Wang (2015). During repeated loadings, these deficiencies can nucleate and form wide crazes already before the desired service life at stress levels far below the nominal yield strength. The second phase is featured by rapid damage growth owing to the coalescence of matured micro-cracks to form large cracks that finally cause material's brittle failure, Ritchie (1999); Lesser (2002); Lemaitre and Desmorat (2005); Lawrimore et al. (2016). However, in many amorphous solids, the timespan of the first phase is invariably orders of magnitude greater than the second phase, Ritchie (1999); 
Janssen et al. (2008); Kanters et al. (2016). Based on that observation, the second, propagation phase is often omitted in the fatigue models.

When dealing with fatigue from low- to high-cycle regimes, a suitable damage rule, in addition to the plasticity model, constitutes a substantive part of the analysis. A multitude of approaches exist for fatigue analysis and can be divided into three main groups: energy, strain, and stress approaches, cf. Bannantine et al. (1990); Ottosen et al. (2008). The stress approach, which has been broadly designed for mechanically governed high-cycle fatigue (HCF), constitutes a backbone of the proposed approach. Abounding of those approaches rely on the fatigue-limit criteria, wherein the fatigue strengths or limits are determined by exploiting a large set of identical cycles. They are also equipped with cumulative damage theories that define the damage increase per cycle, thus requiring that the load spectrum consists of well-defined cycles, Fatemi and Yang (1998); Ottosen et al. (2008). To extract equivalent cycles from load histories, cycle-counting technics are required, Fatemi and Yang (1998). However, it may be challenging to determine a standard cycle from a complex load spectrum, which results in the cycle-counting approaches being inadequate for weighty practical applications.

A different approach to formulate the fatigue model is based on a continuum mechanics framework benefiting an incremental formalism without measuring damage growth per loading cycles, Ottosen et al. (2008); Ayoub et al. (2011); Murakami (2012); Kanters et al. (2016); Holopainen et al. (2017). Such an approach applied here is a modification of an appealing model originally introduced in Ottosen et al. (2008). Albeit the Ottosen et al. (2008) -model is intended for the high-cycle fatigue of metals, its conception is rather general and is suitable for amorphous solids that macroscopically show many similar mechanical and fatigue characteristics, Chaboche (1997); Lesser (2002); Anand and Gurtin (2003); Zaïri et al. (2005); Lugo et al. (2014); Holopainen et al. (2016, 2017). Against canonical $[1-D]$ effective stress damage concepts, cf. Lemaitre and Desmorat (2005); Murakami (2012); Wang et al. (2016), and Holopainen et al. (2017), deformations and stresses are now considered independent on fatigue damage, and damage evolution is formulated by using an endurance surface that shifts in a stress space independent on damage. Owing to this assumption, complex relationship between the deformations and damage as well as challenges in numerical implementation due to a damage-induced stress reduction are not encountered. However, coupling between the fatigue model and the governing constitutive model exists and is treated by using a backstress type of internal variable that explicitly influences both the damage evolution and the plastic flow. The idea is valid for amorphous solids in which the fatigue behavior is ductile, i.e. fatigue damage represents solely the formation of micro-cracks and initial crazing that typically cover most of (over 90\%) the total service life, Janssen et al. (2008); Lugo et al. (2014). In this phase, damaged regions in the material can be considered small and localized compared to those observed after the formation of large cracks. Thus, damage fields do not represent the macroscopic stress reduction in such a manner as is assumed in classical effective stress damage concepts. Since the inhomogeneous stress reduction due to damage is not considered, the 
proposed approach allows for the prediction of fatigue life of an entire structural element by exploiting solely a single location at which the repetitive load spectrum achieves the most determinative fatigue damage.

After introducing kinematics, subsequent thermodynamic treatment as well as the specific fatigue model mentioned in Sections $2.1-2.4$, the approach is demonstrated by introducing specific constitutive equations for technologically important amorphous solid polymers in Section 2.5. The constitutive counterpart of the proposed approach is based on large deformations, i.e. the multiplicative decomposition of the deformation gradient into viscoplastic and elastic parts is applied, cf. Arruda et al. (1993); Anand and Gurtin (2003); Wallin and Holopainen (2012). Many amorphous solids also show viscoelastic deformation behavior, Chaboche (1997); Zä̈ri et al. (2005); Kwang (2016). To improve predictions under cyclic loading processes as well as under long-term creep and recovery, the model is augmented by a viscoelastic element that further splits the elastic part of the deformation gradient into a viscous component and a purely elastic component, Holopainen (2013). When neglecting viscoelasticity and fatigue, the proposed model reduces to the celebrated 8-chain model, referred to as the BPA (Boyce-Parks-Argon) model, see Boyce et al. (1988). Therefore, the presented thermodynamic treatment involving frameindifference, force and moment balances is also valid for the BPA model. By the authors' knowledge, such a treatment in that extent has not previously been presented for the BPA model. The proposed approach including fatigue requires a single parameter set and only few macroscopic quantities, which makes the approach suitable for practical applications.

The article continues by introducing a numerical treatment of the approach mentioned in Section 2.6. Capability of the approach under different load and displacement controlled loadings for technologically important polycarbonate (PC) polymer is addressed. Based on a finite-element implementation, fatigue testing is simulated, and the contribution of an amorphous polymer structure, in view of dispersed inhomogeneities, to the localization of fatigue damage is

debated in Section 3. The article closes with concluding remarks and avenues for future research.

\section{The approach}

\subsection{Kinematics: deformation}

Many amorphous materials exhibit distinct time-dependent behavior and also display nonlinear response during loading and unloading which both repeatedly occur in cyclic fatigue loadings, Lesser (2002); Khan et al. (2006); Dreistadt et al. (2009); Lawrimore et al. (2016). These viscous effects show up as creep and relaxation and are due to a microstructure that needs a relaxation time to attain its equilibrium state after deformation. Representative materials are many polymers that show both the viscoelastic and viscoplastic behavior, Chaboche (1997), Zaïri et al. (2005), and Kwang (2016). Although viscoelastic deformation is considered low in certain amorphous solids including BMGs, Anand and Gurtin (2003), their microstructure also indicates both 
viscoelastic and viscoplastic behavior (decrease in atomic mobility) of which impact inevitably increases under cyclic fatigue processes owing to factors such as ratcheting and temperature rise, Sun and Wang (2015). To ensure the model's ability to follow the viscous deformation behavior under such conditions as well as under creep and recovery, also the elastic deformation is considered viscous, cf. Chaboche (1997); Khan et al. (2006); Dreistadt et al. (2009); Holopainen (2013).

Deformation of a solid body at time $t \in \mathbb{R}^{+}$is defined by the mapping $\boldsymbol{y}: \boldsymbol{X} \mapsto \boldsymbol{x}$ where $\boldsymbol{X} \in \mathfrak{B}$ and $\boldsymbol{x} \in \mathfrak{b}$ are arbitrary material points of the bodies $\mathfrak{B}$ and $\mathfrak{b}$ given in the reference and current deformed placement, respectively.

The Kröner-Lee decomposition is applied, i.e.

$$
\boldsymbol{F}=\boldsymbol{F}^{\mathrm{ve}} \boldsymbol{F}^{\mathrm{p}},
$$

where $\boldsymbol{F}$ is the deformation gradient with Jacobian $J:=\operatorname{det}(\boldsymbol{F})>0$, and $\boldsymbol{F}^{\text {ve }}$ and $\boldsymbol{F}^{\mathrm{p}}$ define the local deformation for the intermediate placement $\overline{\mathcal{N}}$ due to viscoelastic and plastic (also viscous) mechanisms, respectively, see Fig. 1 for clarification. The plastic mechanism denotes the deformation of coiled molecular chains or mutual action of disordered atomic clusters in amorphous polymers and BMGs, respectively, Anand and Gurtin (2003). The viscoelastic mechanism represents deformation of the intermolecular or interatomic amorphous structure. The intermediate placement is only locally defined, i.e. the mappings, $\boldsymbol{F}^{\mathrm{ve}}$ and $\boldsymbol{F}^{\mathrm{p}}$ respectively, are not true deformation gradients. However, both are mappings for which $\operatorname{det}\left(\boldsymbol{F}^{\mathrm{ve}}\right)>0$ and $\operatorname{det}\left(\boldsymbol{F}^{\mathrm{p}}\right)>0$, i.e. the inverses $\boldsymbol{F}^{\mathrm{ve}-1}$ and $\boldsymbol{F}^{\mathrm{p}-1}$ exist. The local, intermediate placement $\overline{\mathcal{N}}$ is stress-free, since it represents the effects of only the plastic deformation uncoupled from elastic deformation. Since $\boldsymbol{F}^{\mathrm{ve}}$ and $\boldsymbol{F}^{\mathrm{p}}$ express elastic and plastic deformation, respectively, they are uncoupled.

The positive definite and symmetric stretch tensor $\boldsymbol{v}$ is defined by the polar decomposition

$$
F=v R,
$$

where $\boldsymbol{R}$ is the rotation tensor.

The plastic deformation in the relaxed intermediate placement is given in terms of a symmetric and positive definite plastic deformation tensor

$$
\overline{\boldsymbol{C}}^{\mathrm{p}}:=\boldsymbol{F}^{\mathrm{p}} \boldsymbol{F}^{\mathrm{p}, \mathrm{T}}
$$

wherein the tensor transpose is symbolized by $\mathrm{T}$. The quantities in the intermediate placement are accentuated by the bar.

The viscoelastic deformation in the current placement is defined according to

$$
\boldsymbol{b}^{\mathrm{ve}}:=\boldsymbol{F}^{\mathrm{ve}} \boldsymbol{F}^{\mathrm{ve}, \mathrm{T}}=\left(\boldsymbol{v}^{\mathrm{ve}}\right)^{2} .
$$

Alternatively, the decomposition

$$
\hat{\boldsymbol{F}}^{\mathrm{p}}=\overline{\boldsymbol{R}} \boldsymbol{F}^{\mathrm{p}} \quad \text { and } \quad \hat{\boldsymbol{F}}^{\mathrm{ve}}=\boldsymbol{F}^{\mathrm{ve}} \overline{\boldsymbol{R}}^{\mathrm{T}},
$$



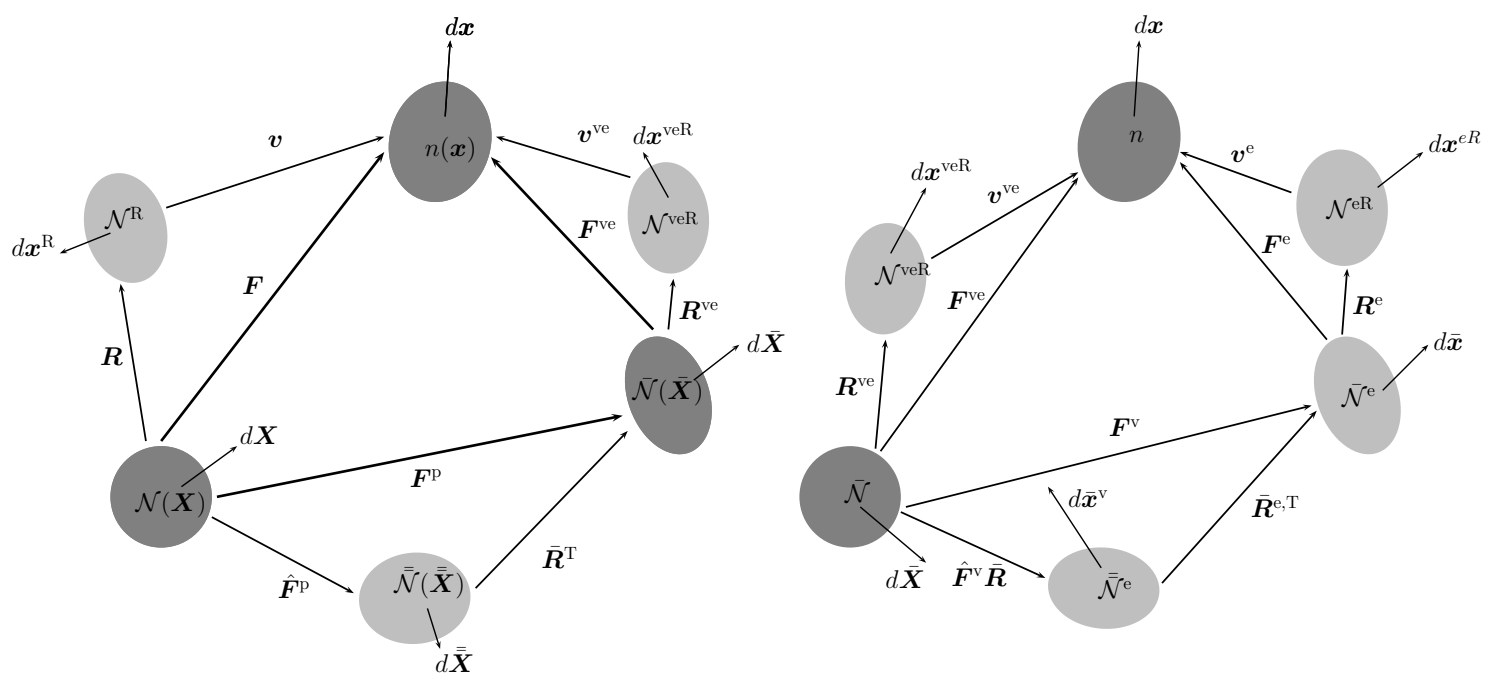

Figure 1: Deformation of a solid body in terms of the mappings between different placements (configurations): $\mathcal{N}, \overline{\mathcal{N}}, n, \overline{\overline{\mathcal{N}}}, \mathcal{N}^{\mathrm{R}}$, and $\mathcal{N}^{\text {ver }}$ for total viscoelastic-viscoplastic deformation (left) and $\overline{\mathcal{N}}^{\mathrm{e}}, \overline{\overline{\mathcal{N}}}^{\mathrm{e}}$, and $\mathcal{N}^{\mathrm{e}}$ for solely viscoelastic deformation split into an elastic (small deformations) and a viscous part (right). The symbols accentuated by the bar(s) refer to intermediate placements.

where $\overline{\boldsymbol{R}}$ is a rotation of the stress-free intermediate placement, can applied in (1). It appears from (5) that the stress-free intermediate placement is not unique and any rigid body rotation leaves it unstressed. Thus, additional restrictions are needed and it will be returned to this issue subsequently in Section 2.6. Fig. 1 illustrates this non-uniqueness, i.e. both $\overline{\mathcal{N}}$ and $\overline{\mathcal{N}}$ represent suitable intermediate configurations.

Based on rubber viscoelasticity Lubliner (1985); Bergström and Boyce (1998); Dal and Kaliske (2009), in accordance with (1), the decomposition of $\boldsymbol{F}^{\mathrm{ve}}$ into a viscous and purely elastic part is applied, i.e.

$$
\boldsymbol{F}^{\mathrm{ve}}=\boldsymbol{F}^{\mathrm{e}} \boldsymbol{F}^{\mathrm{v}}
$$

The decomposition (6) is also known as the Sidoroff decomposition, Sidoroff (1974). The polar decompositions of $\boldsymbol{F}^{\mathrm{e}}$ and $\boldsymbol{F}^{\mathrm{ve}}$ define the positive definite and symmetric stretch tensors $\boldsymbol{v}^{\mathrm{e}}$ and $\boldsymbol{v}^{\mathrm{ve}}$ in the spatial placement as

$$
\boldsymbol{F}^{\mathrm{e}}=\boldsymbol{v}^{\mathrm{e}} \boldsymbol{R}^{\mathrm{e}} \quad \text { and } \quad \boldsymbol{F}^{\mathrm{ve}}=\boldsymbol{v}^{\mathrm{ve}} \boldsymbol{R}^{\mathrm{ve}}
$$

where $\boldsymbol{R}^{\mathrm{e}}$ and $\boldsymbol{R}^{\mathrm{ve}}$ denote the rotation tensors, see Fig. 1 .

Like with (5), also the decomposition

$$
\hat{\boldsymbol{F}}^{\mathrm{ve}}=\hat{\boldsymbol{F}}^{\mathrm{e}} \hat{\boldsymbol{F}}^{\mathrm{v}} \quad \text { where } \quad \hat{\boldsymbol{F}}^{\mathrm{v}}=\overline{\boldsymbol{R}}^{\mathrm{e}} \boldsymbol{F}^{\mathrm{v}} \overline{\boldsymbol{R}}^{\mathrm{T}}, \quad \hat{\boldsymbol{F}}^{\mathrm{e}}=\boldsymbol{F}^{\mathrm{e}} \overline{\boldsymbol{R}}^{\mathrm{e}, \mathrm{T}},
$$

and $\overline{\boldsymbol{R}}^{\text {e }}$ represents a rotation of the elastic intermediate placement, can be applied in (6), see Fig. 1. It then appears from (8) that nor is the elastic intermediate placement unique and certain restrictions are needed, see Section 
$2.6^{1}$.

For later use, symmetric deformation tensors,

$\boldsymbol{b}^{\mathrm{e}}:=\boldsymbol{F}^{\mathrm{e}} \boldsymbol{F}^{\mathrm{e}, \mathrm{T}}=\left(\boldsymbol{v}^{\mathrm{e}}\right)^{2}, \quad \overline{\boldsymbol{b}}^{\mathrm{v}}:=\boldsymbol{F}^{\mathrm{v}} \boldsymbol{F}^{\mathrm{v}, \mathrm{T}}=:\left(\overline{\boldsymbol{v}}^{\mathrm{v}}\right)^{2}, \quad \boldsymbol{b}^{\mathrm{v}}:=\boldsymbol{F}^{\mathrm{e}-\mathrm{T}} \overline{\boldsymbol{b}}^{\mathrm{v}} \boldsymbol{F}^{\mathrm{e}-1}=:\left(\boldsymbol{v}^{\mathrm{v}}\right)^{2}$, $\boldsymbol{c}^{\mathrm{p}}:=\boldsymbol{F}^{\mathrm{ve}-\mathrm{T}} \overline{\boldsymbol{C}}^{\mathrm{p}} \boldsymbol{F}^{\mathrm{ve}-1}$

given in the spatial and the intermediate placements $n$ and $\overline{\mathcal{N}}^{\mathrm{e}}$, are also introduced.

\subsection{Rate kinematics}

Motion of a material body is modeled by the velocity field $\mathbf{v}$, which defines the spatial velocity gradient

$$
\boldsymbol{l}:=\operatorname{grad}(\mathbf{v})=\dot{\boldsymbol{F}} \boldsymbol{F}^{-1}
$$

where the accent dot means the material time derivative. The product decompositions (1) and (6) in (10) yield

$$
\boldsymbol{l}=: \boldsymbol{l}^{\mathrm{ve}}+\boldsymbol{l}^{\mathrm{p}}=\boldsymbol{l}^{\mathrm{e}}+\boldsymbol{l}^{\mathrm{v}}+\boldsymbol{l}^{\mathrm{p}}
$$

wherein

$$
\begin{array}{ll}
\boldsymbol{l}^{\mathrm{e}}:=\dot{\boldsymbol{F}}^{\mathrm{e}} \boldsymbol{F}^{\mathrm{e}-1}, \quad \boldsymbol{l}^{\mathrm{v}}:=\boldsymbol{F}^{\mathrm{e}} \overline{\boldsymbol{l}}^{\mathrm{v}} \boldsymbol{F}^{\mathrm{e}-1}, \quad \boldsymbol{l}^{\mathrm{p}}:=\boldsymbol{F}^{\mathrm{ve}} \overline{\boldsymbol{L}}^{\mathrm{p}} \boldsymbol{F}^{\mathrm{ve}-1}, \\
\overline{\boldsymbol{l}}^{\mathrm{v}}:=\dot{\boldsymbol{F}}^{\mathrm{v}} \boldsymbol{F}^{\mathrm{v}-1}, \quad \overline{\boldsymbol{L}}^{\mathrm{p}}:=\dot{\boldsymbol{F}}^{\mathrm{p}} \boldsymbol{F}^{\mathrm{p}-1} .
\end{array}
$$

Moreover, the symmetric and skew-symmetric counterparts are defined as

$$
\begin{array}{rlrl}
\boldsymbol{d}:=\operatorname{sym}(\boldsymbol{l}), & \boldsymbol{d}^{\mathrm{ve}}:=\operatorname{sym}\left(\boldsymbol{l}^{\mathrm{ve}}\right), & \boldsymbol{d}^{\mathrm{e}}:=\operatorname{sym}\left(\boldsymbol{l}^{\mathrm{e}}\right), \quad \boldsymbol{d}^{\mathrm{v}}:=\operatorname{sym}\left(\boldsymbol{l}^{\mathrm{v}}\right), \\
\overline{\boldsymbol{d}}^{\mathrm{v}}:=\operatorname{sym}\left(\overline{\boldsymbol{l}}^{\mathrm{v}}\right), \quad \boldsymbol{d}^{\mathrm{p}}:=\operatorname{sym}\left(\boldsymbol{l}^{\mathrm{p}}\right), & \overline{\boldsymbol{D}}^{\mathrm{p}}:=\operatorname{sym}\left(\overline{\boldsymbol{L}}^{\mathrm{p}}\right)
\end{array}
$$

and

$$
\begin{aligned}
\boldsymbol{\omega}:=\operatorname{skew}(\boldsymbol{l}), \quad \boldsymbol{\omega}^{\mathrm{ve}}:=\operatorname{skew}\left(\boldsymbol{l}^{\mathrm{ve}}\right), \quad \boldsymbol{\omega}^{\mathrm{e}}:=\operatorname{skew}\left(\boldsymbol{l}^{\mathrm{e}}\right), \quad \boldsymbol{\omega}^{\mathrm{v}}:=\operatorname{skew}\left(\boldsymbol{l}^{\mathrm{v}}\right), \\
\overline{\boldsymbol{\omega}}^{\mathrm{v}}:=\operatorname{skew}\left(\overline{\boldsymbol{l}}^{\mathrm{v}}\right), \quad \boldsymbol{\omega}^{\mathrm{p}}:=\operatorname{skew}\left(\boldsymbol{l}^{\mathrm{p}}\right), \quad \overline{\boldsymbol{W}}^{\mathrm{p}}:=\operatorname{skew}\left(\overline{\boldsymbol{L}}^{\mathrm{p}}\right),
\end{aligned}
$$

respectively.

Based on (3) and (11), the rate of the plastic deformation tensor $\overline{\boldsymbol{C}}^{\mathrm{p}}$ is derived as

$$
\dot{\overline{\boldsymbol{C}}}^{\mathrm{p}}=\overline{\boldsymbol{F}^{\mathrm{p}} \boldsymbol{F}^{\mathrm{p}, \mathrm{T}}}=\dot{\boldsymbol{F}}^{\mathrm{p}} \boldsymbol{F}^{\mathrm{p}, \mathrm{T}}+\boldsymbol{F}^{\mathrm{p}} \dot{\boldsymbol{F}}^{\mathrm{p}, \mathrm{T}}=\overline{\boldsymbol{L}}^{\mathrm{p}} \overline{\boldsymbol{C}}^{\mathrm{p}}+\overline{\boldsymbol{C}}^{\mathrm{p}} \overline{\boldsymbol{L}}^{\mathrm{p}, \mathrm{T}}
$$

In accordance with (15), (9) in conjunction with (12) result in

$$
\dot{\boldsymbol{b}}^{\mathrm{e}}=\boldsymbol{l}^{\mathrm{e}} \boldsymbol{b}^{\mathrm{e}}+\boldsymbol{b}^{\mathrm{e}} \boldsymbol{l}^{\mathrm{e}, \mathrm{T}}, \quad \dot{\overline{\boldsymbol{b}}}^{\mathrm{v}}=\overline{\boldsymbol{l}}^{\mathrm{v}} \overline{\boldsymbol{b}}^{\mathrm{v}}+\overline{\boldsymbol{b}}^{\mathrm{v}} \overline{\boldsymbol{l}}^{\mathrm{v}, \mathrm{T}} .
$$

\footnotetext{
${ }^{1}$ Due to the path dependency, the polar decompositions of $\boldsymbol{F}^{\mathrm{p}}$ and $\boldsymbol{F}^{\mathrm{v}}$ are not applied since it is practically difficult to show the relationship between the rotations and stretching.
} 


\subsection{Thermodynamic treatment of the model}

The theory presented here is based on the principle of virtual power consisting of the following requirements, cf. Anand and Gurtin (2003):

[1] the internal and external virtual powers are equal for all virtual velocities $\mathfrak{V}$ within a subregion or part of the solid body $\mathfrak{A}(t) \subseteq \mathfrak{b}(t)$, i.e. $\mathfrak{W}^{\text {ext }}(\mathfrak{A}, \mathfrak{V})=\mathfrak{W}^{\text {int }}(\mathfrak{A}, \mathfrak{V})$ (power balance);

[2] given any part $\mathfrak{A}$ and any virtual velocity $\mathfrak{V}$, the internal power $\mathfrak{W}^{\text {int }}(\mathfrak{A}, \mathfrak{V})$ is frame-indifferent, i.e. invariant under all changes in frame.

\section{Macroscopic force balance}

Based on the power balance [1] mentioned above, one is at liberty to choose a virtual velocity field $\tilde{\mathfrak{V}}$ consistent with (10), i.e. in the current placement. Denoting outward unit vector of the boundary region $\partial \mathfrak{A}$ of the part $\mathfrak{A}$ by $\boldsymbol{n}$,

$$
\mathfrak{W}^{\text {ext }}(\mathfrak{A}, \mathfrak{V})=\int_{\partial \mathfrak{A}} \boldsymbol{t}(\boldsymbol{n}) \cdot \tilde{\mathbf{v}} d a+\int_{\mathfrak{A}} \boldsymbol{f} \cdot \tilde{\mathbf{v}} d v=\int_{\mathfrak{A}} \boldsymbol{\tau} \cdot \operatorname{grad}(\tilde{\mathbf{v}}) d v
$$

where $\boldsymbol{t}(\boldsymbol{n})$ and $\boldsymbol{f}$ are a local surface traction and a body force, respectively; $\tilde{\mathbf{v}}$ is a virtual velocity field of $\mathfrak{V}$; and $\boldsymbol{\tau}$ is the governing stress field. Using the divergence theorem, (17) takes the form

$$
\int_{\partial \mathfrak{A}}(\boldsymbol{t}(\boldsymbol{n})-\boldsymbol{\tau} \boldsymbol{n}) \cdot \tilde{\mathbf{v}} d a+\int_{\mathfrak{A}}(\operatorname{div}(\boldsymbol{\tau})+\boldsymbol{f}) \cdot \tilde{\mathbf{v}} d v=0
$$

The relationship (18) must hold for all $\mathfrak{A}$ and $\tilde{\mathbf{v}}$ and thus, $\boldsymbol{t}(\boldsymbol{n})-\boldsymbol{\tau} \boldsymbol{n}=\mathbf{0}$ and $\operatorname{div}(\boldsymbol{\tau})+\boldsymbol{f}=\mathbf{0}$, which equalities represent the macroscopic force balances.

\section{Microforce balance}

Assuming the internal virtual power is expended by a stress $\tau$ rate conjugate to $\tilde{\boldsymbol{l}}^{\mathrm{ve}}$, and also by an internal microstress $\boldsymbol{\tau}^{\mathrm{p}}$ rate conjugate to $\tilde{\boldsymbol{l}}^{\mathrm{p}}$, i.e.

$$
\mathfrak{W}^{\text {int }}(\mathfrak{A}, \mathfrak{V}):=\int_{\mathfrak{A}}\left(\boldsymbol{\tau}: \tilde{\boldsymbol{l}}^{\mathrm{e}}+\boldsymbol{\tau}: \tilde{\boldsymbol{l}}^{\mathrm{v}}+\boldsymbol{\tau}^{\mathrm{p}}: \tilde{\boldsymbol{l}}^{\mathrm{p}}\right) d v,
$$

cf. Anand and Gurtin (2003). The operator : in (19) is argued to be the tensor trace as $\boldsymbol{A}: \boldsymbol{B}:=\operatorname{trace}\left(\boldsymbol{A} \boldsymbol{B}^{\mathrm{T}}\right)$.

Assuming the virtual velocity field, $\tilde{\mathbf{v}}$ in (17) vanishes. Consequently, the external virtual power identically vanishes when the internal virtual power also vanishes according to the power balance [1]. Then, by (10) and (11), $\tilde{\boldsymbol{l}}^{\mathrm{e}}+\tilde{\boldsymbol{l}}^{\mathrm{v}}=$ $-\tilde{\boldsymbol{l}}^{\mathrm{p}}$ when (19) becomes

$$
\left(\boldsymbol{\tau}^{\mathrm{p}}-\boldsymbol{\tau}\right): \tilde{\boldsymbol{l}}^{\mathrm{p}}=0 .
$$

Since $\tilde{\boldsymbol{l}}^{\mathrm{p}}$ is an arbitrary tensor field, (20) is fulfilled by assuming $\boldsymbol{\tau}^{\mathrm{p}}=\boldsymbol{\tau}$. The stress $\tau^{\mathrm{p}}$ represents an internal microstress affected by damage due to mutual interaction of microcavities, see Lemaitre and Desmorat (2005). Damage prior to the cracking phase, as considered here, is strongly localized and thus does not influence the macroscopic stress. 


\section{Frame-indifference}

Considering an arbitrary change in frame of the (Euclidean) space through which the material body shifts. In the current frame, $\mathfrak{A}$ transforms rigidly to a region $\mathfrak{A}^{*}, \mathfrak{V}$ to a virtual velocity $\mathfrak{V}^{*}$, and $\boldsymbol{\tau}$ to a stress $\boldsymbol{\tau}^{*}$ conjugate to virtual velocities. The theory must be invariant under such transformations, i.e. under a motion of a homogeneous body of the form

$$
\boldsymbol{y}(\boldsymbol{X}, t) \rightarrow \boldsymbol{Q}(t)(\boldsymbol{y}(\boldsymbol{X}, t)-\boldsymbol{O})+\boldsymbol{q}(t)
$$

where $\boldsymbol{Q}(t)$ is an orthogonal rotation tensor, $\boldsymbol{q}(t)$ is a vector at time $t$, and $\boldsymbol{O}$ is a fixed origin.

Considering that the reference placement is unaltered under the choice of changes in frame, while the current and the intermediate placements are dependent on such choices,

$$
\begin{aligned}
\boldsymbol{F} & \rightarrow \boldsymbol{Q F}, \quad \boldsymbol{F}^{\mathrm{p}} \rightarrow \overline{\boldsymbol{Q}} \boldsymbol{F}^{\mathrm{p}}, \quad \boldsymbol{F}^{\mathrm{ve}} \rightarrow \boldsymbol{Q} \boldsymbol{F}^{\mathrm{ve}} \overline{\boldsymbol{Q}}^{\mathrm{T}}, \\
\boldsymbol{F}^{\mathrm{e}} & \rightarrow \boldsymbol{Q} \boldsymbol{F}^{\mathrm{e}} \overline{\boldsymbol{Q}}^{\mathrm{e}, \mathrm{T}}, \quad \boldsymbol{F}^{\mathrm{v}} \rightarrow \overline{\boldsymbol{Q}}^{\mathrm{e}} \boldsymbol{F}^{\mathrm{v}} \overline{\boldsymbol{Q}}^{\mathrm{T}}
\end{aligned}
$$

where a distinction is made between the rotations of the placements. Furthermore, the deformation measures in (3) and (9) become

$$
\begin{aligned}
\overline{\boldsymbol{C}}^{\mathrm{p}} & \rightarrow \overline{\boldsymbol{Q}} \overline{\boldsymbol{C}}^{\mathrm{p}} \overline{\boldsymbol{Q}}^{\mathrm{T}}, \quad \boldsymbol{b}^{\mathrm{ve}} \rightarrow \boldsymbol{Q} \boldsymbol{b}^{\mathrm{ve}} \boldsymbol{Q}^{\mathrm{T}}, \\
\boldsymbol{b}^{\mathrm{e}} & \rightarrow \boldsymbol{Q} \boldsymbol{b}^{\mathrm{e}} \boldsymbol{Q}^{\mathrm{T}}, \quad \overline{\boldsymbol{b}}^{\mathrm{v}} \rightarrow \overline{\boldsymbol{Q}}^{\mathrm{e}} \overline{\boldsymbol{b}}^{\mathrm{v}} \overline{\boldsymbol{Q}}^{\mathrm{e}, \mathrm{T}}, \\
\boldsymbol{b}^{\mathrm{v}} & \rightarrow \boldsymbol{Q} \boldsymbol{F}^{\mathrm{e}-\mathrm{T}} \overline{\boldsymbol{Q}}^{\mathrm{e}, \mathrm{T}} \overline{\boldsymbol{Q}}^{\mathrm{e}} \overline{\boldsymbol{b}}^{\mathrm{v}} \overline{\boldsymbol{Q}}^{\mathrm{e}, \mathrm{T}} \overline{\boldsymbol{Q}}^{\mathrm{e}} \boldsymbol{F}^{\mathrm{e}-1} \boldsymbol{Q}^{\mathrm{T}}=\boldsymbol{Q} \boldsymbol{b}^{\mathrm{v}} \boldsymbol{Q}^{\mathrm{T}} .
\end{aligned}
$$

Based on the transformations in (22), the motion of a body transforms according to

$$
\begin{aligned}
& \boldsymbol{l} \rightarrow \boldsymbol{Q} \boldsymbol{l} \boldsymbol{Q}^{\mathrm{T}}+\dot{\boldsymbol{Q}} \boldsymbol{Q}^{\mathrm{T}}, \quad \boldsymbol{d} \rightarrow \boldsymbol{Q} \boldsymbol{d} \boldsymbol{Q}^{\mathrm{T}}, \\
& \overline{\boldsymbol{L}}^{\mathrm{p}} \rightarrow \overline{\boldsymbol{Q}} \overline{\boldsymbol{L}}^{\mathrm{p}} \overline{\boldsymbol{Q}}^{\mathrm{T}}+\dot{\overline{\boldsymbol{Q}}} \overline{\boldsymbol{Q}}^{\mathrm{T}}, \quad \overline{\boldsymbol{D}}^{\mathrm{p}} \rightarrow \overline{\boldsymbol{Q}} \overline{\boldsymbol{D}}^{\mathrm{p}} \overline{\boldsymbol{Q}}^{\mathrm{T}}, \\
& \boldsymbol{l}^{\mathrm{p}} \rightarrow \boldsymbol{Q} \boldsymbol{l}^{\mathrm{p}} \boldsymbol{Q}^{\mathrm{T}}+\boldsymbol{Q} \boldsymbol{F}^{\mathrm{ve}} \overline{\boldsymbol{Q}}^{\mathrm{T}} \dot{\overline{\boldsymbol{Q}}} \boldsymbol{F}^{\mathrm{ve}-1} \boldsymbol{Q}^{\mathrm{T}}, \\
& \boldsymbol{l}^{\mathrm{ve}} \rightarrow \boldsymbol{Q} \boldsymbol{l}^{\mathrm{ve}} \boldsymbol{Q}^{\mathrm{T}}+\dot{\boldsymbol{Q}} \boldsymbol{Q}^{\mathrm{T}}+\boldsymbol{Q} \boldsymbol{F}^{\mathrm{ve}} \dot{\overline{\boldsymbol{Q}}}^{\mathrm{T}} \overline{\boldsymbol{Q}} \boldsymbol{F}^{\mathrm{ve}-1} \boldsymbol{Q}^{\mathrm{T}}, \\
& \boldsymbol{l}^{\mathrm{e}} \rightarrow \boldsymbol{Q} \boldsymbol{l}^{\mathrm{e}} \boldsymbol{Q}^{\mathrm{T}}+\dot{\boldsymbol{Q}} \boldsymbol{Q}^{\mathrm{T}}+\boldsymbol{Q} \boldsymbol{F}^{\mathrm{e}} \dot{\overline{\boldsymbol{Q}}}^{\mathrm{e}, \mathrm{T}} \overline{\boldsymbol{Q}}^{\mathrm{e}} \boldsymbol{F}^{\mathrm{e}-1} \boldsymbol{Q}^{\mathrm{T}}, \\
& \overline{\boldsymbol{l}}^{\mathrm{v}} \rightarrow \overline{\boldsymbol{Q}}^{\mathrm{e}} \overline{\boldsymbol{l}}^{\mathrm{v}} \overline{\boldsymbol{Q}}^{\mathrm{e}, \mathrm{T}}+\dot{\overline{\boldsymbol{Q}}}^{\mathrm{e}} \overline{\boldsymbol{Q}}^{\mathrm{e}, \mathrm{T}}+\overline{\boldsymbol{Q}}^{\mathrm{e}} \boldsymbol{F}^{\mathrm{v}} \dot{\overline{\boldsymbol{Q}}}^{\mathrm{T}} \overline{\boldsymbol{Q}} \boldsymbol{F}^{\mathrm{v}-1} \overline{\boldsymbol{Q}}^{\mathrm{e}, \mathrm{T}}, \\
& \boldsymbol{l}^{\mathrm{v}} \rightarrow \boldsymbol{Q} \boldsymbol{l}^{\mathrm{v}} \boldsymbol{Q}^{\mathrm{T}}+\boldsymbol{Q} \boldsymbol{F}^{\mathrm{e}} \overline{\boldsymbol{Q}}^{\mathrm{e}, \mathrm{T}} \dot{\overline{\boldsymbol{Q}}}^{\mathrm{e}} \boldsymbol{F}^{\mathrm{e}-1} \boldsymbol{Q}^{\mathrm{T}}+\boldsymbol{Q} \boldsymbol{F}^{\mathrm{e}} \boldsymbol{F}^{\mathrm{v}} \dot{\overline{\boldsymbol{Q}}}^{\mathrm{T}} \overline{\boldsymbol{Q}} \boldsymbol{F}^{\mathrm{v}-1} \boldsymbol{F}^{\mathrm{e}-1} \boldsymbol{Q}^{\mathrm{T}} \\
& =\boldsymbol{Q} \boldsymbol{l}^{\mathrm{v}} \boldsymbol{Q}^{\mathrm{T}}-\boldsymbol{Q} \boldsymbol{F}^{\mathrm{e}} \dot{\overline{\boldsymbol{Q}}}^{\mathrm{e}, \mathrm{T}} \overline{\boldsymbol{Q}}^{\mathrm{e}} \boldsymbol{F}^{\mathrm{e}-1} \boldsymbol{Q}^{\mathrm{T}}-\boldsymbol{Q} \boldsymbol{F}^{\mathrm{ve}} \overline{\boldsymbol{Q}}^{\mathrm{T}} \dot{\overline{\boldsymbol{Q}}} \boldsymbol{F}^{\mathrm{ve}-1} \boldsymbol{Q}^{\mathrm{T}} .
\end{aligned}
$$

The results in (24) yield $\boldsymbol{l}=\boldsymbol{l}^{\mathrm{e}}+\boldsymbol{l}^{\mathrm{v}}+\boldsymbol{l}^{\mathrm{p}} \rightarrow \boldsymbol{Q}\left(\boldsymbol{l}^{\mathrm{e}}+\boldsymbol{l}^{\mathrm{v}}+\boldsymbol{l}^{\mathrm{p}}\right) \boldsymbol{Q}^{\mathrm{T}}+\dot{\boldsymbol{Q}} \boldsymbol{Q}^{\mathrm{T}}=$ $\boldsymbol{Q} \boldsymbol{l} \boldsymbol{Q}^{\mathrm{T}}+\dot{\boldsymbol{Q}} \boldsymbol{Q}^{\mathrm{T}}, \boldsymbol{\omega}=\boldsymbol{Q} \boldsymbol{\omega} \boldsymbol{Q}^{\mathrm{T}}+\dot{\boldsymbol{Q}} \boldsymbol{Q}^{\mathrm{T}}$, and $\overline{\boldsymbol{W}}^{\mathrm{p}} \rightarrow \overline{\boldsymbol{Q}} \overline{\boldsymbol{W}}^{\mathrm{p}} \overline{\boldsymbol{Q}}^{\mathrm{T}}+\dot{\overline{\boldsymbol{Q}}} \overline{\boldsymbol{Q}}^{\mathrm{T}}$.

It is assumed that the fields of a virtual velocity transform as their nonvirtual counterparts do. Then, the frame-indifference [2] indicates that $\mathfrak{W}^{\text {int }}(\mathfrak{A}, \mathfrak{V})=$ $\mathfrak{W}^{\text {int* }}\left(\mathfrak{A}^{*}, \mathfrak{V}^{*}\right)$, i.e. considering the micro-force balance, (19) and (20),

$$
\mathfrak{W}^{\text {int* }}\left(\mathfrak{A}^{*}, \mathfrak{V}^{*}\right):=\int_{\mathfrak{A}}\left(\boldsymbol{\tau}^{*}:\left(\boldsymbol{Q} \tilde{\boldsymbol{l}} \boldsymbol{Q}^{\mathrm{T}}+\dot{\boldsymbol{Q}} \boldsymbol{Q}^{\mathrm{T}}\right) d v .\right.
$$


Since $\mathfrak{A}$, the change in frame, the field $\tilde{\boldsymbol{l}}$, and the skew-symmetric tensor $\dot{Q} Q^{\mathrm{T}}$ are all arbitrary, the frame-indifference [2] results in the stress transforming according to

$$
\tau \rightarrow Q \tau Q^{\mathrm{T}}
$$

and is symmetric, i.e. $\tau=\tau^{\mathrm{T}}$ similar to the Kirchhoff stress (macroscopic moment balance).

Lemma 2.1. The results in (24) dictate the invariance of the internal power according to the requirement [2].

Proof. Using the transformations (24) and (26), and the symmetry of $\boldsymbol{\tau}$ (macroscopic moment balance) yield

$$
\begin{aligned}
& \boldsymbol{\tau}: \boldsymbol{d}^{\mathrm{p}} \rightarrow \boldsymbol{\tau}: \boldsymbol{d}^{\mathrm{p}}+\overline{\boldsymbol{T}}: \overline{\boldsymbol{Q}}^{\mathrm{T}} \dot{\overline{\boldsymbol{Q}}}=\boldsymbol{\tau}: \boldsymbol{d}^{\mathrm{p}} \\
& \boldsymbol{\tau}: \boldsymbol{d}^{\mathrm{e}} \rightarrow \boldsymbol{\tau}:\left(\boldsymbol{d}^{\mathrm{e}}+\dot{\boldsymbol{Q}} \boldsymbol{Q}^{\mathrm{T}}\right)+\overline{\boldsymbol{\tau}}^{\mathrm{e}}: \dot{\overline{\boldsymbol{Q}}}^{\mathrm{e}, \mathrm{T}} \overline{\boldsymbol{Q}}^{\mathrm{e}}=\boldsymbol{\tau}: \boldsymbol{d}^{\mathrm{e}}, \\
& \boldsymbol{\tau}: \boldsymbol{d}^{\mathrm{v}} \rightarrow \boldsymbol{\tau}: \boldsymbol{d}^{\mathrm{v}}+\overline{\boldsymbol{\tau}}^{\mathrm{e}}: \overline{\boldsymbol{Q}}^{\mathrm{e}, \mathrm{T}} \dot{\overline{\boldsymbol{Q}}}^{\mathrm{e}}+\overline{\boldsymbol{T}}: \dot{\overline{\boldsymbol{Q}}}^{\mathrm{T}} \overline{\boldsymbol{Q}}=\boldsymbol{\tau}: \boldsymbol{d}^{\mathrm{v}}
\end{aligned}
$$

where $\overline{\boldsymbol{T}}$ and $\overline{\boldsymbol{\tau}}^{\mathrm{e}}$ are symmetric, mixed-variant counterparts of $\boldsymbol{\tau}$ expressed in the stress-free and elastic intermediate placements, respectively. Exploiting the rule $\boldsymbol{A}: \boldsymbol{B}=\boldsymbol{A}^{\mathrm{T}}: \boldsymbol{B}^{\mathrm{T}}$ for all second order tensors $\boldsymbol{A}, \boldsymbol{B}$, the stresses are defined as

$$
\mathbf{0}=\boldsymbol{\tau}: \boldsymbol{F}^{\mathrm{ve}} \overline{\boldsymbol{Q}}^{\mathrm{T}} \dot{\overline{\boldsymbol{Q}}} \boldsymbol{F}^{\mathrm{ve}-1}-\boldsymbol{\tau}: \boldsymbol{F}^{\mathrm{ve}-\mathrm{T}} \dot{\overline{\boldsymbol{Q}}}^{\mathrm{T}} \overline{\boldsymbol{Q}} \boldsymbol{F}^{\mathrm{ve}, \mathrm{T}}=: \overline{\boldsymbol{T}}: \overline{\boldsymbol{Q}}^{\mathrm{T}} \dot{\overline{\boldsymbol{Q}}}
$$

and

$$
\mathbf{0}=\boldsymbol{\tau}: \boldsymbol{F}^{\mathrm{e}} \dot{\overline{\boldsymbol{Q}}}^{\mathrm{e}, \mathrm{T}} \overline{\boldsymbol{Q}}^{\mathrm{e}} \boldsymbol{F}^{\mathrm{e}-1}-\boldsymbol{\tau}: \boldsymbol{F}^{\mathrm{e}-\mathrm{T}} \dot{\overline{\boldsymbol{Q}}}^{\mathrm{e}, \mathrm{T}} \overline{\boldsymbol{Q}}^{\mathrm{e}} \boldsymbol{F}^{\mathrm{e}, \mathrm{T}}=\overline{\boldsymbol{\tau}}^{\mathrm{e}}: \dot{\overline{\boldsymbol{Q}}}^{\mathrm{e}, \mathrm{T}} \overline{\boldsymbol{Q}}^{\mathrm{e}}
$$

\section{Dissipation power}

Mechanical theory based on the second law of thermodynamics in its spatial form takes the form

$$
\int_{\mathfrak{A}} \dot{\varphi} d v \leq \mathfrak{W}^{\text {ext }}(\mathfrak{A})=\mathfrak{W}^{\text {int }}(\mathfrak{A})
$$

where $\dot{\varphi}$ is the rate of the thermomechanical potential $\varphi$. The formula (28) is known as a dissipation inequality.

Since the part $\mathfrak{A}$ of the solid body is arbitrary, the second thermodynamics principle (28) can be localized. Exploiting the equations (11), (13), and (20), (28) takes the following form of the so-called Clausius-Duhem inequality:

$$
\mathcal{D}=\boldsymbol{\tau}: \boldsymbol{d}^{\mathrm{e}}+\boldsymbol{\tau}: \boldsymbol{d}^{\mathrm{v}}+\boldsymbol{\tau}: \boldsymbol{d}^{\mathrm{p}}-\dot{\varphi} \geq 0,
$$

in which $\mathcal{D}$ denotes the power of the local dissipation in the deformed current placement. 


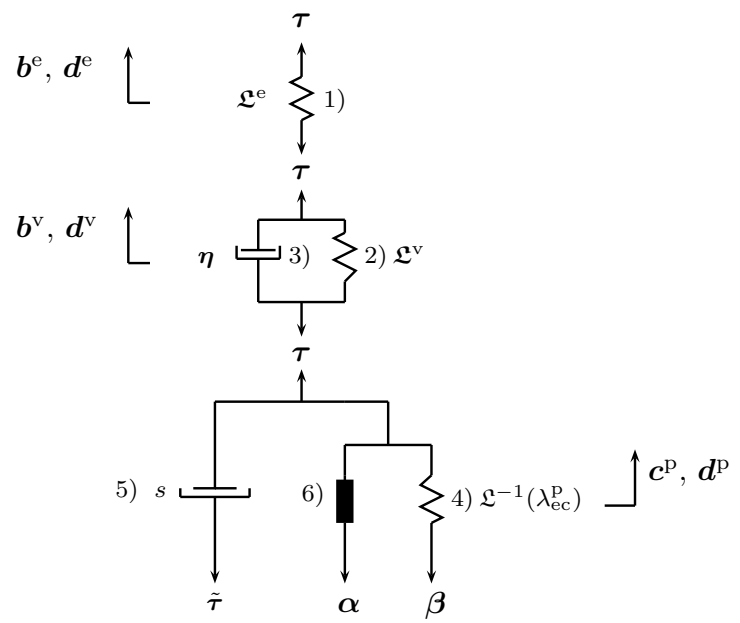

Figure 2: Rheological description of the approach comprising of elastic springs 1) and 2 ), a viscoelastic dashpot 3), a nonlinear spring 4), a viscoplastic dashpot 5), and a nonlinear device 6) for fatigue. Difference between the backstresses $\boldsymbol{\beta}+\boldsymbol{\alpha}$ and the Kirchhoff stress $\boldsymbol{\tau}$ determines the driving stress $\tilde{\boldsymbol{\tau}}$.

\section{Constitutive theory}

The constitutive restrictions that have been required so far are rather universal. The approach is constrained by imposing additional constitutive constraints based on existing theories and knowledge on viscoelastic-viscoplastic amorphous materials, Khan et al. (2006); Dreistadt et al. (2009); Holopainen (2013); Sun and Wang (2015). The applied constitutive model seizes creep and recovery through the Kelvin-Voigt element which is comprised of an elastic spring 2) and a viscous dashpot 3) as demonstrated in Fig. 2. Its function with the elastic spring 1 ) is able to predict the stress relaxation. This model was extended to large deformations in Haward and Thackray (1968). Another Kelvin-Voigt-like element consists of a viscoplastic dashpot 5) parallel to a spring 4) resulting in anisotropic nature of material at large strains. A nonlinear device 6) dictates the fatigue development.

When neglecting viscoelasticity and fatigue, the proposed model reduces to the well-known BPA model for amorphous polymers including the elements 1), 4), and 5), see Boyce et al. (1988), and thus, the given thermodynamic treatment is valid for the BPA model too. A detailed account concerning the extended BPA model including the first Kelvin-Voigt element for viscoelasticity is discussed in (Holopainen, 2013, Sections 4-4.1).

Due to the difficulties of a single potential to define different phenomena of viscoelasticity, viscoplasticity, and damage well, see Chaboche (1997), a broader framework based on several independent potentials is proposed. Therefore, the Helmholtz free energy $\varphi$, consistent with the proposed concept (stresses are independent on damage), is taken to be a sum of a viscoelastic, a viscoplastic, 
and a damage part as

$$
\begin{aligned}
& \varphi=\hat{\varphi}\left(\boldsymbol{b}^{\mathrm{e}}, \overline{\boldsymbol{b}}^{\mathrm{v}}, \overline{\boldsymbol{C}}^{\mathrm{p}}, D ; \varphi^{\alpha}\right)=\hat{\varphi}^{\mathrm{e}}\left(\boldsymbol{b}^{\mathrm{e}}\right)+\hat{\varphi}^{\mathrm{v}}\left(\overline{\boldsymbol{b}}^{\mathrm{v}}\right)+\hat{\varphi}^{\mathrm{p}}\left(\overline{\boldsymbol{C}}^{\mathrm{p}}\right)+ \\
& \hat{\varphi}^{\mathrm{d}}(D)+\hat{\varphi}^{\alpha}\left(\boldsymbol{b}^{\mathrm{e}}, \boldsymbol{c}^{\mathrm{p}}\right)
\end{aligned}
$$

where the potentials $\varphi^{\mathrm{e}}, \varphi^{\mathrm{v}}, \varphi^{\mathrm{p}}, \varphi^{\mathrm{d}}$, and $\varphi^{\alpha}$ are contributions owing to the elastic spring 1 , the spring 2 , the Langevin spring 4 , pure damage, and the device 6 , respectively, shown in Fig. 2. Since the damage development represents the formation of micro-cracks that typically covers most of (over $90 \%$ ) of the total fatigue life, Janssen et al. (2008); Lugo et al. (2014), damaged regions in the material are considered small and localized, and stresses (and deformations) are practically independent on damage. Therefore, damage does not enter the first three functions in (30). However, the damage variable $D$ is considered as a mesoscopic quantity that affects separately the free energy, i.e. the dissipation power (29) includes the effect of material degradation during thermodynamic processes, cf. Chaboche (1997). Moreover, a coupling between the constitutive and fatigue model exists and is treated by the function $\varphi^{\alpha}$ defined subsequently.

Remark 2.2. Expressions for the potential functions in (30) are, as defined subsequently, functions of their tensor arguments defined solely via the invariants of the arguments. This requirement guarantees that the free energy remains unchanged under rigid body rotations.

The rate $\dot{\varphi}$ becomes

$$
\dot{\varphi}=\frac{\partial \varphi^{\mathrm{e}}}{\partial \boldsymbol{b}^{\mathrm{e}}}: \dot{\boldsymbol{b}}^{\mathrm{e}}+\frac{\partial \varphi^{\mathrm{v}}}{\partial \overline{\boldsymbol{b}}^{\mathrm{v}}}: \dot{\overline{\boldsymbol{b}}}^{\mathrm{v}}+\frac{\partial \varphi^{\mathrm{p}}}{\partial \overline{\boldsymbol{C}}^{\mathrm{p}}}: \dot{\overline{\boldsymbol{C}}}^{\mathrm{p}}+\frac{\partial \varphi^{\mathrm{d}}}{\partial D} \dot{D}+\dot{\varphi}^{\alpha}
$$

where

$$
\dot{\varphi}^{\alpha}:=\boldsymbol{\alpha}: \boldsymbol{d}^{\mathrm{p}} .
$$

was introduced. The tensor-valued quantity $\boldsymbol{\alpha}=\hat{\boldsymbol{\alpha}}\left(\boldsymbol{b}^{\mathrm{e}}, \boldsymbol{c}^{\mathrm{p}}\right)$ will be defined as a backstress that drives fatigue and also influences the development of plastic deformation, see Fig. 2. Then, the term (32) in (31) constitutes the coupling between the constitutive and fatigue models. Since degradation of the material occurs in plasticized zones, cf. e.g. Li et al. (1995); Janssen et al. (2008); Lugo et al. (2014); Ding et al. (2017), the plastic rate of deformation $\boldsymbol{d}^{\mathrm{p}}$ is taken to be the rate conjugate of $\boldsymbol{\alpha}$. Evolution of $\boldsymbol{\alpha}$ and $\boldsymbol{d}^{\mathrm{p}}$ will be discussed later on.

Taking advantage of (9) and (16), and noting the symmetry of $\partial \varphi^{\mathrm{e}} / \partial \boldsymbol{b}^{\mathrm{e}}$ results in

$$
\frac{\partial \varphi^{\mathrm{e}}}{\partial \boldsymbol{b}^{\mathrm{e}}}: \dot{\boldsymbol{b}}^{\mathrm{e}}=2 \frac{\partial \varphi^{\mathrm{e}}}{\partial \boldsymbol{b}^{\mathrm{e}}} \boldsymbol{b}^{\mathrm{e}}: \boldsymbol{d}^{\mathrm{e}}
$$

In accordance with (33),

$$
\frac{\partial \varphi^{\mathrm{v}}}{\partial \overline{\boldsymbol{b}}^{\mathrm{v}}}: \dot{\overline{\boldsymbol{b}}}^{\mathrm{v}}=: 2 \frac{\partial \varphi^{\mathrm{v}}}{\partial \overline{\boldsymbol{b}}^{\mathrm{v}}} \overline{\boldsymbol{b}}^{\mathrm{v}}: \overline{\boldsymbol{d}}^{\mathrm{v}}=: 2 \boldsymbol{F}^{\mathrm{e}}\left(\frac{\partial \varphi^{\mathrm{v}}}{\partial \overline{\boldsymbol{b}}^{\mathrm{v}}} \overline{\boldsymbol{b}}^{\mathrm{v}}\right) \boldsymbol{F}^{\mathrm{e}, \mathrm{T}}: \boldsymbol{d}^{\mathrm{v}}
$$

and

$$
\frac{\partial \varphi^{\mathrm{p}}}{\partial \overline{\boldsymbol{C}}^{\mathrm{p}}}: \dot{\overline{\boldsymbol{C}}}^{\mathrm{p}}=: 2 \frac{\partial \varphi^{\mathrm{p}}}{\partial \overline{\boldsymbol{C}}^{\mathrm{p}}} \overline{\boldsymbol{C}}^{\mathrm{p}}: \overline{\boldsymbol{D}}^{\mathrm{p}}=: \overline{\boldsymbol{B}}: \overline{\boldsymbol{D}}^{\mathrm{p}}
$$


in which $\overline{\boldsymbol{B}}$ represents a backstress due to the plastic work being reserved in the material. Its counterpart $\boldsymbol{\beta}$ given in the deformed current placement becomes

$$
\boldsymbol{\beta}=\boldsymbol{F}^{\mathrm{ve}} \overline{\boldsymbol{B}} \boldsymbol{F}^{\mathrm{ve}, \mathrm{T}},
$$

see Boyce et al. $(1988,1989)$.

A complementary dissipation potential, $\phi$, is defined by the viscoelastic, viscoplastic, and the damage components as

$$
\phi\left(\boldsymbol{\tau}^{\mathrm{ve}}, \tilde{\boldsymbol{\tau}}, Y\right):=\phi^{\mathrm{ve}}\left(\boldsymbol{\tau}^{\mathrm{ve}}\right)+\phi^{\mathrm{vp}}(\tilde{\boldsymbol{\tau}})+\phi^{\mathrm{d}}(Y)
$$

where $\boldsymbol{\tau}^{\mathrm{ve}}$ and $\tilde{\boldsymbol{\tau}}$ stand for driving stresses in the dashpot 3) and 4), see Fig. 2 , and $Y$ is a driving force for material degradation. Exploiting the proposed dissipation potential, the dissipation power is defined as

$$
\mathcal{D}=\frac{\partial \phi^{\mathrm{ve}}}{\partial \boldsymbol{\tau}^{\mathrm{ve}}}: \boldsymbol{\tau}^{\mathrm{ve}}+\frac{\partial \phi^{\mathrm{vp}}}{\partial \tilde{\boldsymbol{\tau}}}: \tilde{\boldsymbol{\tau}}+\frac{\partial \phi^{\mathrm{d}}}{\partial Y} Y
$$

Substituting (32), (33), (34), and (35) into (29) and taking (37) into account yield

$$
\begin{aligned}
& \left(\boldsymbol{\tau}-2 \frac{\partial \varphi^{\mathrm{e}}}{\partial \boldsymbol{b}^{\mathrm{e}}} \boldsymbol{b}^{\mathrm{e}}\right): \boldsymbol{d}^{\mathrm{e}}+\left(\boldsymbol{\tau}-2 \boldsymbol{F}^{\mathrm{e}}\left(\frac{\partial \varphi^{\mathrm{v}}}{\partial \overline{\boldsymbol{b}}^{\mathrm{v}}} \overline{\boldsymbol{b}}^{\mathrm{v}}\right) \boldsymbol{F}^{\mathrm{e}, \mathrm{T}}\right): \boldsymbol{d}^{\mathrm{v}}-\frac{\partial \phi^{\mathrm{ve}}}{\partial \boldsymbol{\tau}^{\mathrm{ve}}}: \boldsymbol{\tau}^{\mathrm{ve}}+ \\
& (\boldsymbol{\tau}-\boldsymbol{\beta}-\boldsymbol{\alpha}): \boldsymbol{d}^{\mathrm{p}}-\frac{\partial \phi^{\mathrm{vp}}(\tilde{\boldsymbol{\tau}})}{\partial \tilde{\boldsymbol{\tau}}}: \tilde{\boldsymbol{\tau}}-\left(\frac{\partial \varphi^{\mathrm{d}}}{\partial D} \dot{D}+\frac{\partial \phi^{\mathrm{d}}}{\partial Y} Y\right)=0 .
\end{aligned}
$$

Since equation (38) must fulfill all possible thermodynamically admissible processes, the constitutive equations

$$
\boldsymbol{\tau}=2 \frac{\partial \varphi^{\mathrm{e}}}{\partial \boldsymbol{b}^{\mathrm{e}}} \boldsymbol{b}^{\mathrm{e}}
$$

and

$$
\dot{D}=\frac{\partial \phi^{\mathrm{d}}}{\partial Y}
$$

with the additional equations,

$$
\boldsymbol{\tau}: \boldsymbol{d}^{\mathrm{v}}=2 \boldsymbol{F}^{\mathrm{e}}\left(\frac{\partial \varphi^{\mathrm{v}}}{\partial \overline{\boldsymbol{b}}^{\mathrm{v}}} \overline{\boldsymbol{b}}^{\mathrm{v}}\right) \boldsymbol{F}^{\mathrm{e}, \mathrm{T}}: \boldsymbol{d}^{\mathrm{v}}+\frac{\partial \phi^{\mathrm{ve}}}{\partial \boldsymbol{\tau}^{\mathrm{ve}}}: \boldsymbol{\tau}^{\mathrm{ve}},
$$

and

$$
(\boldsymbol{\tau}-\boldsymbol{\beta}-\boldsymbol{\alpha}): \boldsymbol{d}^{\mathrm{p}}=: \tilde{\boldsymbol{\tau}}: \boldsymbol{d}^{\mathrm{p}}=\frac{\partial \phi^{\mathrm{vp}}(\tilde{\boldsymbol{\tau}})}{\partial \tilde{\boldsymbol{\tau}}}: \tilde{\boldsymbol{\tau}}=:-\dot{s},
$$

are obtained. When obtaining (40), the relationship

$$
Y:=-\partial \varphi^{\mathrm{d}} / \partial D
$$

was defined. 
Flow rule

Considering many amorphous solids, against many metals, there does not exist a threshold yield stress value below which only elastic and viscoelastic deformations occur, i.e. the plastic deformation develops once a loading is applied, see Boyce et al. (1988); Arruda et al. (1993). Based on this characteristic, the current rate of plastic deformation, $\boldsymbol{d}^{\mathrm{p}}$, is determined to align with the normalized direction of the effective stress $\tilde{\boldsymbol{\tau}}^{\text {dev }}$ given in (42) as

$$
\boldsymbol{d}^{\mathrm{p}}:=\dot{\gamma}^{\mathrm{p}} \boldsymbol{n}, \quad \boldsymbol{n}:=\frac{\tilde{\boldsymbol{\tau}}^{\mathrm{dev}}}{\sqrt{2} \tau}, \quad \tau:=\sqrt{\frac{1}{2} \tilde{\boldsymbol{\tau}}^{\mathrm{dev}}: \tilde{\boldsymbol{\tau}}^{\mathrm{dev}}}
$$

wherein the notation dev implies to the deviatoric part being defined by the identity $\boldsymbol{i}$ as $[\cdot]^{\mathrm{dev}}:=[\cdot]-1 / 3 \operatorname{trace}([\cdot]) \boldsymbol{i}$ for each second order tensor [.]. It then follows that

$$
2 J^{\mathrm{p}} \dot{J}^{\mathrm{p}}:=\frac{d}{d t} \operatorname{det}\left(\overline{\boldsymbol{C}}^{\mathrm{p}}\right)=\operatorname{trace}\left(\overline{\boldsymbol{C}}^{\mathrm{p}-\mathrm{T}} \dot{\overline{\boldsymbol{C}}}^{\mathrm{p}, \mathrm{T}}\right)=\operatorname{trace}\left(\overline{\boldsymbol{D}}^{\mathrm{p}}\right)=\operatorname{trace}\left(\boldsymbol{d}^{\mathrm{p}}\right)=0,
$$

i.e. the plastic deformation is incompressible and thus, $J=J^{\mathrm{ve}}:=\operatorname{det}\left(\boldsymbol{F}^{\mathrm{ve}}\right)$.

The flow rule influenced by both damage and the backstress $\beta$ is generally known from the Gurson model, which model has later been augmented e.g. by Anand and Gearing (2004); Zaïri et al. (2011) for amorphous polymers in large deformations. The difference between these previous models and the proposed model for fatigue is that damage does not explicitly affect the flow rule, but through the backstress $\boldsymbol{\alpha}$ involved in the effective stress. As has previously been discussed, this feature of the proposed approach originates from the presumption that deformations and stresses during the crack initiation phase are independent on damage.

\section{Viscoelastic deformation}

The applied stress $\boldsymbol{\tau}$ is independent of fatigue damage $D$, in which sense it can be termed an effective stress in accordance with Lemaitre and Desmorat (2005); Murakami (2012). The molecular chain structure of polymers and atomic structure of BMGs are presumed to be completely disordered when the following isotropic strain energy functions,

$$
\varphi^{\mathrm{e}}=\frac{1}{2} \kappa^{\mathrm{e}}\left(I_{1}^{\mathrm{e}}\right)^{2}+2 \mu^{\mathrm{e}} J_{2}^{\mathrm{e}}, \varphi^{\mathrm{v}}=\frac{1}{2} \kappa^{\mathrm{v}}\left(I_{1}^{\mathrm{v}}\right)^{2}+2 \mu^{\mathrm{v}} J_{2}^{\mathrm{v}},
$$

where $\kappa^{\mathrm{e}}, \kappa^{\mathrm{v}}, \mu^{\mathrm{e}}$, and $\mu^{\mathrm{v}}$ are the bulk and the shear moduli, respectively, are used. The logarithmic invariants present in (45) are

$$
\begin{aligned}
& I_{1}^{\mathrm{e}}:=\operatorname{trace}\left(\ln \boldsymbol{v}^{\mathrm{e}}\right)=\ln J^{\mathrm{e}}, I_{1}^{\mathrm{v}}:=\operatorname{trace}\left(\ln \boldsymbol{v}^{\mathrm{v}}\right)=\ln J^{\mathrm{v}}, \\
& J_{2}^{\mathrm{e}}:=1 / 2\left(\ln \boldsymbol{v}^{\mathrm{e}}\right)^{\operatorname{dev}}:\left(\ln \boldsymbol{v}^{\mathrm{e}}\right)^{\operatorname{dev}}, J_{2}^{\mathrm{v}}:=1 / 2\left(\ln \boldsymbol{v}^{\mathrm{v}}\right)^{\operatorname{dev}}:\left(\ln \boldsymbol{v}^{\mathrm{v}}\right)^{\operatorname{dev}}
\end{aligned}
$$

where $J^{\mathrm{e}}:=\operatorname{det}\left(\boldsymbol{v}^{\mathrm{e}}\right)$ and $J^{\mathrm{v}}:=\operatorname{det}\left(\boldsymbol{v}^{\mathrm{v}}\right)$.

The first component in the dissipation potential (37) is defined as

$$
\phi^{\mathrm{ve}}:=1 / 2 \boldsymbol{\tau}^{\mathrm{ve}}: \boldsymbol{\eta}^{-1}: \boldsymbol{\tau}^{\mathrm{ve}}
$$


in which a symmetric fourth-order tensor $\boldsymbol{\eta}$, for simplicity, is considered a constant. In addition, assuming $\boldsymbol{\tau}^{\mathrm{ve}}:=\boldsymbol{\eta}: \boldsymbol{d}^{\mathrm{v}}$ it follows from (39), (41), (45), and (46) that the constitutive law is based on the stress equilibrium

$$
\boldsymbol{\tau}=\mathfrak{L}^{\mathrm{e}}: \ln \boldsymbol{v}^{\mathrm{e}}=\boldsymbol{\eta}: \boldsymbol{d}^{\mathrm{v}}+\mathfrak{L}^{\mathrm{v}}: \ln \boldsymbol{v}^{\mathrm{v}}
$$

where $\mathfrak{L}^{\mathrm{e}}$ and $\mathfrak{L}^{\mathrm{v}}$ stand for the standard fourth order stiffness tensors, for instance, $\mathfrak{L}^{\mathrm{e}}:=2 \mu^{\mathrm{e}}\left(\mathfrak{I}+\left(3 \kappa^{\mathrm{e}}-2 \mu^{\mathrm{e}}\right) /\left(6 \mu^{\mathrm{e}}\right) \boldsymbol{i} \otimes \boldsymbol{i}\right)$ where the tensor $\mathfrak{I}$ denotes the fourth order, spatial identity. In following treatments, the shear and the bulk moduli will be given in terms of the Young's modulus and the Poisson's ratio.

The viscous damper in (47) is defined by

$$
\boldsymbol{\eta}=\eta_{1}(\mathfrak{I}-1 / 3 \boldsymbol{i} \otimes \boldsymbol{i})+\eta_{2} \boldsymbol{i} \otimes \boldsymbol{i}
$$

in which the viscosities $\eta_{1}$ and $\eta_{2}$ govern elastic shear and volumetric deformation, see Reese and Govinjee (1998). The symbol $\otimes$ denotes the common tensor product which defines the components of $\boldsymbol{\eta}$ in orthonormal coordinates as

$$
\boldsymbol{\eta}_{i j k l}=\eta_{1}\left(\delta_{i k} \delta_{j l}+\delta_{i l} \delta_{j k}-2 / 3 \delta_{i j} \delta_{k l}\right) / 2+\eta_{2} \delta_{i j} \delta_{k l} .
$$

Using a more simple form, as given in Boyce et al. (1989); Reese and Govinjee (1998), the latter term in (48) and (49) is considered to vanish, i.e. $\eta_{2}=0$. It then follows that solely a few new material parameters, $E^{\mathrm{v}}, \nu^{\mathrm{v}}$, and $\eta_{1}:=\eta$, are introduced to the proposed model extension for viscoelasticity.

\section{Damage contribution}

In the isotropic damage mechanics framework based on (40), the damage contribution of the dissipation potential is constrained. During fatigue damage processes, the part of free energy $\varphi^{\mathrm{d}}$ needed for a damage increase tends to decrease, which in agreement with experimental observations that embodies a remarkable material decay before complete failure, see e.g. Murakami (2012). Since damage never decreases, it is positive and an assumption that the driving force $Y$ for damage is, according to (43), also positive, builds up this fatigue characteristic.

Theorem 2.3. Assuming the force $Y$ is positive, the proposed model satisfies the Clausius-Duhem inequality, i.e. the power of the local dissipation, $\mathcal{D}$, is greater than or equal to zero.

Proof. Taking into account the equations (39), (41), (43), (44), and (46) in (38), the Clausius-Duhem inequality (29) takes the form

$$
\mathcal{D}=\boldsymbol{d}^{\mathrm{v}}: \boldsymbol{\eta}: \boldsymbol{d}^{\mathrm{v}}+\sqrt{2} \tau \dot{\gamma}^{\mathrm{p}}+\dot{D} Y \geq 0 .
$$

Since $\dot{\gamma}^{\mathrm{p}} \geq 0$ and $\dot{D} \geq 0$ (damage never decreases) in (50), the dissipation power is always positive or zero provided $Y \geq 0$ 


\subsection{Fatigue model}

The applied fatigue model can be considered as an evolution equation-based fatigue model where the endurance surface is defined to be a function of a stress history. The proposed model is an annex of an appealing HCF model proposed by Ottosen et al. (2008), suitable for predicting mechanically dominated fatigue of metals. Although this approach is aimed for use in the context of metal fatigue, essentially characterized by dislocation micromechanisms, its formulation is rather general and suitable for amorphous solids that macroscopically show many similar fatigue characteristics, Lesser (2002); Anand and Gurtin (2003); Lugo et al. (2014); Holopainen et al. (2017). The most important characteristic is the macroscopical asymptotical extremes of lifetimes, i.e. the endurance limits can be determined and fatigue under these limits is attenuated, Lugo et al. (2014).

According to the original model by Ottosen etal (2008), it is the backstress $\alpha$ of the effective stress that defines the midpoint of the endurance surface and also germinates the shift of the endurance surface. This idea is followed here. A thermodynamically consistent effective stress is given by (42). The effective stress further comprises the backstress $\boldsymbol{\beta}$ since the plastic deformation, against many metals, develops once a loading is applied and may be significant affecting hardening (especially around inhomogeneities). Furthermore, degradation of the material occurs in plasticized zones, cf. e.g. Li et al. (1995); Janssen et al. (2008); Lugo et al. (2014); Holopainen et al. (2017). A consequence is that the proposed fatigue model is suitable for application in the LCF regime, where a significant macroscopic plastic deformation may take place. The applied fatigue model is described in detail in (Holopainen et al., 2017, Sec. 2.4) and is therefore only briefly summarized below.

According to this model, the endurance function is defined as

$$
\beta=\left(\bar{\tau}+a I_{1}-\sigma_{0}\right) / \sigma_{0}
$$

wherein

$$
\bar{\tau}:=\sqrt{\frac{3}{2} \tilde{\tau}^{\mathrm{dev}}: \tilde{\boldsymbol{\tau}}^{\mathrm{dev}}}:=\sqrt{3 J_{2}\left(\boldsymbol{\tau}^{\mathrm{dev}}-\boldsymbol{\beta}^{\mathrm{dev}}-\boldsymbol{\alpha}\right)} .
$$

In the endurance function, the first stress invariant $I_{1}=\operatorname{trace}(\boldsymbol{\tau})$ reflects the effect of the hydrostatic stress, $I_{1} / 3$, i.e. the hydrostatic tension promotes the fatigue accumulation whereas fatigue is attenuated under hydrostatic compression. The positive parameter $a$ in (51) defines in uniaxial cyclic loading the (negative) slope of the Haigh-diagram, and $\sigma_{0}$ denotes the endurance limit for zero mean stress at infinite fatigue life. The endurance surface, as demonstrated in Fig. 3, is spherical in the deviatoric plane, and it is the $\boldsymbol{\alpha}+\boldsymbol{\beta}^{\text {dev }}$ tensor that determines the midpoint ${ }^{2}$.

\footnotetext{
${ }^{2}$ Considering many metals with an explicit yield surface. If the midpoints of their endurance surface and the yield surface would be separately defined by $\boldsymbol{\alpha}$ and $\boldsymbol{\beta}$, respectively, these could locate at the sites where they do not cross or are not one inside another as they probably should in order to provide realistic results.
} 
As has been previously mentioned, fatigue failure occurs in plasticized zones and the backstress $\boldsymbol{\alpha}$ that drives fatigue is also presumed to evolve with plastic deformation. Although the plastic deformation may be low under service loadings at room temperature, its influence on microstructural changes and also on macroscopic material response can be significant due to ratcheting and temperature rise, Anand and Gurtin (2003); Janssen et al. (2008); Lugo et al. (2014); Holopainen et al. (2017). The plastic deformation under such conditions may result in strain hardening and anisotropy of the material represented by the backstress $\boldsymbol{\beta}$. Therefore, the evolution of $\boldsymbol{\alpha}$ is described by

$$
\boldsymbol{\alpha}^{\nabla}=C\left(\boldsymbol{\tau}^{\mathrm{dev}}-\boldsymbol{\beta}^{\mathrm{dev}}-\boldsymbol{\alpha}\right) \dot{\beta}-\boldsymbol{\beta}^{\mathrm{dev}, \nabla}
$$

where $C$ is a material parameter and

$$
\boldsymbol{\beta}^{\nabla}=\dot{\boldsymbol{\beta}}-\boldsymbol{l} \boldsymbol{\beta}-\boldsymbol{\beta} \boldsymbol{l}^{\mathrm{T}}
$$

defines an objective rate of $\boldsymbol{\beta}$. Hydrostatic stress effects also the evolution of $\boldsymbol{\alpha}$ through the rate $\dot{\beta}$ of the endurance surface (51).

Once a fatigue loading is actuated, the endurance surface is able to track the current stress due to the movement of $\boldsymbol{\alpha}+\boldsymbol{\beta}^{\mathrm{dev}}$ that is, according to (53), always in the direction of $\boldsymbol{\tau}^{\mathrm{dev}}-\boldsymbol{\beta}^{\mathrm{dev}}-\boldsymbol{\alpha}$, see Fig. 3. The evolution equation (53) also reveals that the $\boldsymbol{\alpha}$ tensor can memorize the load history since its evolution allows the value $\beta=0$, i.e. the movement of the endurance surface in the stress space. It is only postulated that fatigue damage $D$ and the backstress $\boldsymbol{\alpha}$, which is considered an overall force for $D$, solely evolve on or outside the endurance surface $(\beta \geq 0)$ and only when $\dot{\beta}>0$, i.e. when the stress has crossed the surface and recedes from it, cf. Ottosen et al. (2008). This situation is demonstrated in Fig. 3.

Proposition 2.4. The fatigue damage only develops under the condition

$$
\beta \geq 0, \quad \dot{\beta}>0 \Rightarrow \dot{D}>0, \quad \boldsymbol{\alpha}^{\nabla} \neq \mathbf{0} .
$$

What remains to be shown is that $\boldsymbol{\alpha}$ and its rate $\boldsymbol{\alpha}^{\nabla}$ are objective.

Corollary 2.5. Backstress $\boldsymbol{\alpha}$ and its rate $\boldsymbol{\alpha}^{\nabla}$ are objective, i.e. they fulfill the second requirement of the principle of virtual power.

Proof. Based on the moment balance the spatial deviatoric Kirchhoff stress $\boldsymbol{\tau}^{\text {dev }}$ is symmetric and transforms by $\boldsymbol{\tau}^{\text {dev }} \rightarrow \boldsymbol{Q} \boldsymbol{\tau}^{\text {dev }} \boldsymbol{Q}^{\mathrm{T}}$, cf. (26). Moreover, using the transformations (22) in conjunction with (35) and (36) yields $\boldsymbol{\beta}^{\text {dev }} \rightarrow$ $\boldsymbol{Q} \boldsymbol{\beta}^{\mathrm{dev}} \boldsymbol{Q}^{\mathrm{T}}$. Additionally, assuming that $\boldsymbol{\alpha}$ (symmetric, given in the current placement) transforms under a change in frame (observer) as $\boldsymbol{\alpha} \rightarrow \boldsymbol{Q} \boldsymbol{\alpha} \boldsymbol{Q}^{\mathrm{T}}$, it follows that

$$
\begin{aligned}
\boldsymbol{\alpha}^{\nabla} \rightarrow & C\left(\boldsymbol{Q} \boldsymbol{\tau}^{\mathrm{dev}} \boldsymbol{Q}^{\mathrm{T}}-\boldsymbol{Q} \boldsymbol{\beta}^{\mathrm{dev}} \boldsymbol{Q}^{\mathrm{T}}-\boldsymbol{Q} \boldsymbol{\alpha} \boldsymbol{Q}^{\mathrm{T}}\right) \dot{\beta}^{*}-\dot{\boldsymbol{Q}} \boldsymbol{\beta}^{\mathrm{dev}} \boldsymbol{Q}^{\mathrm{T}}-\boldsymbol{Q} \dot{\boldsymbol{\beta}}^{\mathrm{dev}} \boldsymbol{Q}^{\mathrm{T}}-\boldsymbol{Q} \boldsymbol{\beta}^{\mathrm{dev}} \dot{\boldsymbol{Q}}^{\mathrm{T}}+ \\
& \boldsymbol{Q} \boldsymbol{l} \boldsymbol{Q}^{\mathrm{T}} \boldsymbol{Q} \boldsymbol{\beta}^{\mathrm{dev}} \boldsymbol{Q}^{\mathrm{T}}+\dot{\boldsymbol{Q}} \boldsymbol{Q}^{\mathrm{T}} \boldsymbol{Q} \boldsymbol{\beta}^{\mathrm{dev}} \boldsymbol{Q}^{\mathrm{T}}+\boldsymbol{Q} \boldsymbol{\beta}^{\mathrm{dev}} \boldsymbol{Q}^{\mathrm{T}} \boldsymbol{Q} \boldsymbol{l}^{\mathrm{T}} \boldsymbol{Q}^{\mathrm{T}}+\boldsymbol{Q} \boldsymbol{\beta}^{\mathrm{dev}} \boldsymbol{Q}^{\mathrm{T}} \boldsymbol{Q} \dot{\boldsymbol{Q}}^{\mathrm{T}} \\
= & \boldsymbol{Q} C\left(\boldsymbol{\tau}^{\mathrm{dev}}-\boldsymbol{\beta}^{\mathrm{dev}}-\boldsymbol{\alpha}\right) \dot{\beta}^{*} \boldsymbol{Q}^{\mathrm{T}}-\boldsymbol{Q}\left(\dot{\boldsymbol{\beta}}^{\mathrm{dev}}-\boldsymbol{l} \boldsymbol{\beta}^{\mathrm{dev}}-\boldsymbol{\beta}^{\mathrm{dev}} \boldsymbol{l}^{\mathrm{T}}\right) \boldsymbol{Q}^{\mathrm{T}}=\boldsymbol{Q} \boldsymbol{\alpha} \boldsymbol{Q}^{\mathrm{T}},
\end{aligned}
$$




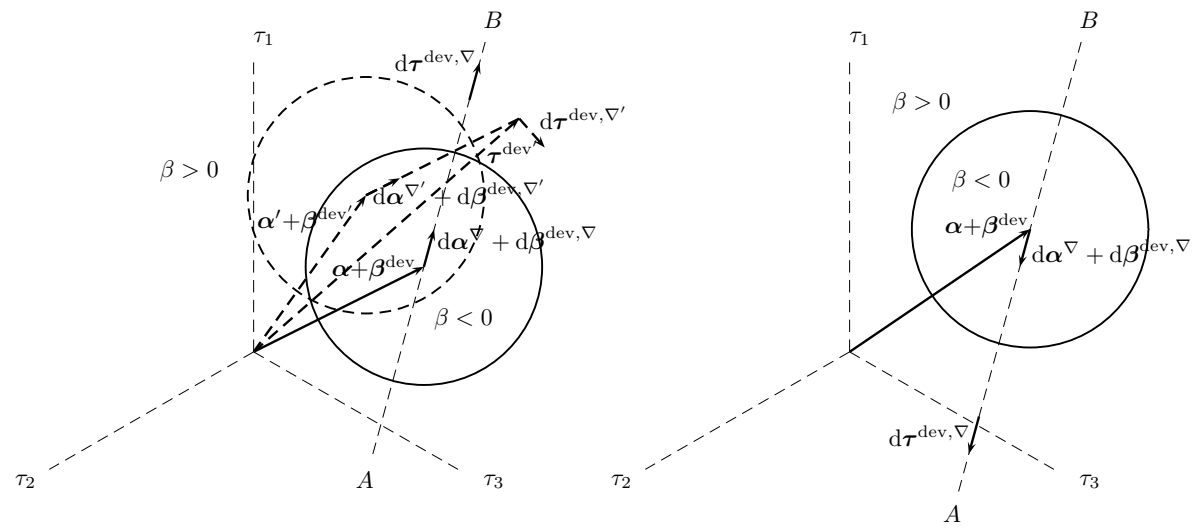

Figure 3: Movement of an endurance surface in deviatoric plane under a fluctuating stress (possibly non-proportional) (left). The endurance surface reaches the current stress point and then starts to move between the states $A$ and $B$ (not necessarily fixed) (right). Peripheries of the surfaces in an initial and the current state are highlighted by the dashed and solid curves, respectively. Marking d signifies a small increment, and the symbol $\nabla$ refers to objective stress rates. Reused with permission from Elsevier license.

i.e. $\alpha^{\nabla}$ is objective since $\beta$ is a scalar-valued function for which $\dot{\beta}^{*}=\dot{\beta}$ holds, see (51). In addition to the objectivity of $\boldsymbol{\tau}$ and $\boldsymbol{\beta}$, it appears that $\boldsymbol{\beta}^{\nabla}$ is objective. In turn, the assumption that $\boldsymbol{\alpha}^{\nabla}$ is objective reveals that $\boldsymbol{\alpha}$ in (53) is also objective

\section{Damage evolution}

When dealing with fatigue from low to high cycles, a suitable damage rule, in addition to the plasticity model, constitutes an integral part of the analysis. The damage growth is computed by using an evolution law based on a scalar valued damage quantity. Description of damage by a scalar valued quantity is valid since damage now represents solely the formation of micro-cracks that, most of all, typically covers a majority of the total fatigue life of amorphous solids (over 90\%), Marissen et al. (2001); Janssen et al. (2008); Lugo et al. (2014).

Let then $x_{0}, x_{\mathrm{c}}, t_{0}$, and $t_{\mathrm{c}}$ be the initial material placement, final critical placement, initial time, and critical time instant, respectively. Then, damage at an initial instant and a local fatigue failure instantaneously prior to a rapid progress of small cracks to a form of large cracks are given by $D=D\left(x_{0}, t_{0}\right):=$ $D_{0} \geq 0$ and $D\left(x_{\mathrm{c}}, t_{\mathrm{c}}\right)=D_{\mathrm{c}}=1$, respectively.

Based on (40) and Proposition 2.4, an exponential expression,

$$
\dot{D}=K \exp \left(f\left(\beta ; L_{1}, L_{2}, \vartheta\right)\right) \dot{\beta}
$$

where $K, \vartheta, L_{1}$, and $L_{2}$ are positive parameters, will be applied for the damage evolution, see the Appendix A. Since damage never decreases, (56) implies that $\dot{\beta} \geq 0$. The damage evolution (56) obviously satisfies the dissipation inequality $\mathcal{D} \geq 0$, see Theorem 2.3. 
Many amorphous polymers exhibit only a minor accumulation of the applied stress as the cyclic lifetime decreases, see the Wöhler curves in Janssen et al. (2008). The behavior of BMGs is considerably different; the endurance limit may be remarkable lower than the fatigue strength under LCF loadings, Launeya et al. (2009). To capture different behaviors, a function $f$ having two distinct linear asymptotes for a $\beta$-function is defined, i.e.

$$
f\left(\beta ; L_{1}, L_{2}, \vartheta\right):=\left[L_{1}-L_{2}\left(1+\frac{L_{2}}{\vartheta \beta}\left(\exp \left(-\vartheta \beta / L_{2}\right)-1\right)\right)\right] \beta .
$$

The function $f$ has the asymptote $L_{1} \beta$ as $\beta$ is near zero in the HCF-regime and $\left(L_{1}-L_{2}\right) \beta$ as $\beta$ is large in the LCF-regime. The curvature $\vartheta$ completes the definition of how rapidly the second asymptote is reached.

\subsection{Specific constitutive equations}

In this section, the approach is refined to concern the mechanical behavior of technologically important amorphous polymers under ambient conditions at room temperature.

\section{Plastic deformation}

The plastic deformation is managed by the plastic shear strain rate

$$
\dot{\gamma}^{\mathrm{p}}=\dot{\gamma}_{0} \exp \left(-\frac{A s_{\mathrm{s}}}{T}\left(1-\left(\frac{\tilde{\tau}^{\mathrm{dev}}}{J^{\mathrm{ve}} s_{\mathrm{s}}}\right)^{\frac{5}{6}}\right)\right)
$$

that describes an isotropic barrier to chain segment rotation followed by strain softening. The effective stress $\tilde{\tau}$ is defined by (42) and $\dot{\gamma}_{0}, A$, and $T$ are positive parameters, see Boyce et al. (1989); Holopainen (2013). The athermal shear strength is given by $s_{\mathrm{s}}=s_{1}+\alpha p$ in which $p=-\operatorname{trace}(\boldsymbol{\sigma}) / 3$ is the pressure (in terms of the Cauchy stress $\boldsymbol{\sigma}=\boldsymbol{\tau} / J)$ and $\alpha$ is a pressure dependence factor.

Function of the viscoplastic dashpot for strain softening is modeled by

$$
\dot{s}_{1}=h_{1} \dot{\gamma}^{\mathrm{p}}\left(1-s_{1} / s_{\mathrm{ss}}\right), \quad s_{1}(0)=s_{0}>s_{\mathrm{ss}},
$$

where the positive parameters $h_{1}$ and $s_{\mathrm{ss}}$ define the softening slope and the saturation value of $s_{1}$, i.e. $s_{1}>s_{\mathrm{ss}}$ as $\dot{s}_{1}<0$. Despite the fact that $-\dot{s}_{1} \neq-\dot{s}=$ $\sqrt{2} \tau \dot{\gamma}^{\mathrm{p}} \geq 0$ in (42), the rate $\dot{s}_{1}$ also satisfies the dissipation inequality (50).

The plastic part of the free energy is based on non-Gaussian chain statistics and is governed by the following function:

$$
\varphi^{\mathrm{p}}\left(\lambda_{\mathrm{ec}}^{\mathrm{p}}\right)=C_{R}\left(\frac{\lambda_{\mathrm{ec}}^{\mathrm{p}}}{\sqrt{N}} \chi+\ln \left(\frac{\chi}{\sinh (\chi)}\right)\right) \geq 0, \quad \varphi^{\mathrm{p}}(1)=0
$$

where $C_{R}\left(\left[C_{R}\right]=\mathrm{MPa}\right)$ and $N$ are material parameters and $\chi:=\mathfrak{L}^{-1}\left(\lambda_{\mathrm{ec}}^{\mathrm{p}} / \sqrt{N}\right)$ is the inverse of the Langevin function $\mathfrak{L}$, Boyce et al. (1988). Using a non-affine plastic network stretch,

$$
\lambda_{\mathrm{ec}}^{\mathrm{p}}:=\frac{1}{\sqrt{3}} \sqrt{\operatorname{trace}\left(\overline{\boldsymbol{C}}^{\mathrm{p}}\right)},
$$


the backstress $\boldsymbol{\beta}$ introduced in (35) takes the following form

$$
\overline{\boldsymbol{B}}=\frac{C^{\mathrm{R}}}{3 \lambda_{\mathrm{ec}}^{\mathrm{p}}} \sqrt{N} \chi \overline{\boldsymbol{C}}^{\mathrm{p}}
$$

When evaluating $\chi$ in (62), the Padé approximation is used, Wallin and Holopainen (2012); Holopainen (2013).

\subsection{Numerical analysis of the approach}

\section{Uniqueness of the intermediate placements}

To recall from (5) and (8), as a consequence of the multiplicative decompositions (1) and (6), the arbitrariness of the intermediate placements can be given as

$$
\boldsymbol{F}=\boldsymbol{F}^{\mathrm{ve}} \overline{\boldsymbol{R}}^{\mathrm{T}} \overline{\boldsymbol{R}} \boldsymbol{F}^{\mathrm{p}}=: \hat{\boldsymbol{F}}^{\mathrm{ve}} \hat{\boldsymbol{F}}^{\mathrm{p}}, \quad \hat{\boldsymbol{F}}^{\mathrm{ve}}=\boldsymbol{F}^{\mathrm{e}} \overline{\boldsymbol{R}}^{\mathrm{e}, \mathrm{T}}\left(\overline{\boldsymbol{R}}^{\mathrm{e}} \boldsymbol{F}^{\mathrm{v}} \overline{\boldsymbol{R}}^{\mathrm{T}}\right)=: \hat{\boldsymbol{F}}^{\mathrm{e}} \hat{\boldsymbol{F}}^{\mathrm{v}} .
$$

It appears that the rotations $\overline{\boldsymbol{R}}$ and $\overline{\boldsymbol{R}}^{\mathrm{e}}$, which determine the orientations of the intermediate placements $\overline{\mathcal{N}}$ and $\overline{\mathcal{N}}^{\mathrm{e}}$, are not unique and additional kinematic assumptions are needed. Although the plastic spin in certain finite strain elasto-plasticity models has been chosen to vanish, see e.g. Miehe et al. (1993); Anand and Gurtin (2003), the BPA model involving nonzero plastic spin is strictly followed here. The assumption of a nonzero plastic spin is motivated by the observations showing the elastic portion of deformation is small especially when compared to the plastic deformation, see Agah-Tehrani et al. (1987); Boyce et al. (1988, 1989); Arruda et al. (1993); Dreistadt et al. (2009). The reason is that the plastic deformation, against many metals, develops rapidly once the loading is applied. Therefore, the elastic counterpart $\hat{\boldsymbol{F}}^{\mathrm{e}}$ in (63) is considered symmetric ${ }^{3}$, which restriction constraints the rotation $\overline{\boldsymbol{R}}^{\mathrm{e}}$ :

$$
\hat{\boldsymbol{F}}^{\mathrm{ve}}=\boldsymbol{F}^{\mathrm{ve}} \overline{\boldsymbol{R}}^{\mathrm{T}}=\boldsymbol{v}^{\mathrm{ve}} \boldsymbol{R}^{\mathrm{ve}} \overline{\boldsymbol{R}}^{\mathrm{T}}=\operatorname{sym}\left(\hat{\boldsymbol{F}}^{\mathrm{e}}\right) \overline{\boldsymbol{F}}^{\mathrm{v}}=\operatorname{sym}\left(\boldsymbol{v}^{\mathrm{e}} \boldsymbol{R}^{\mathrm{e}} \overline{\boldsymbol{R}}^{\mathrm{e}, \mathrm{T}}\right)\left(\overline{\boldsymbol{R}}^{\mathrm{e}} \boldsymbol{F}^{\mathrm{v}} \overline{\boldsymbol{R}}^{\mathrm{T}}\right) .
$$

Moreover, the presumption that $\hat{\boldsymbol{F}}^{\mathrm{ve}}$ in the decomposition (63) is symmetric at the end of the integration interval is applied. Then, through an appropriate choice of rotations, $\hat{\boldsymbol{F}}^{\mathrm{e}}=\boldsymbol{v}^{\mathrm{e}}, \hat{\boldsymbol{F}}^{\mathrm{ve}}=\boldsymbol{v}^{\mathrm{ve}}$, and the decomposition (64) reduces to

$$
\boldsymbol{v}^{\mathrm{ve}}=\boldsymbol{v}^{\mathrm{e}} \hat{\boldsymbol{F}}^{\mathrm{v}}=\boldsymbol{v}^{\mathrm{e}}\left(\boldsymbol{R}^{\mathrm{e}} \boldsymbol{F}^{\mathrm{v}} \boldsymbol{R}^{\mathrm{ve}, \mathrm{T}}\right) .
$$

Noting the symmetry of $\boldsymbol{v}^{\mathrm{ve}}$, a possible choice is that $\hat{\boldsymbol{F}}^{\mathrm{v}}$ is symmetric, i.e. $\boldsymbol{v}^{\mathrm{e}}$ and $\hat{\boldsymbol{F}}^{\mathrm{v}}$ are coaxial. The restriction that the elastic deformation gradient is symmetric and specifies uniqueness of the stress-free intermediate placement of elasticviscoplastic responses is known from Boyce et al. (1988); Weber and Anand (1990); Arruda et al. (1993). In contrast, the identification of the intermediate placements by assuming $\boldsymbol{v}^{\mathrm{e}}$ and $\hat{\boldsymbol{F}}^{\mathrm{v}}$ to be coaxial (rotation $\boldsymbol{R}^{\mathrm{ve}}$ is restricted)

\footnotetext{
${ }^{3}$ In more detail, $\hat{\boldsymbol{F}}^{\mathrm{e}}$ is a mapping between two configurations when only the presentation of the component matrix is symmetric: $\left[\hat{\boldsymbol{F}}^{\mathrm{e}}\right]_{. J}^{i}=\left[\hat{\boldsymbol{F}}^{\mathrm{e}}\right]_{. i}^{J}$.
} 
in (65) can be questioned. However, there exists experimental evidence that it is the viscoplastic deformation that encompasses a majority of the deformation (including rotations), also when long-term creep and recovery take place, Arruda et al. (1993); Melick (2003); Dreistadt et al. (2009). Due to this reason, the identification of the intermediate placements by the proposed constraints is accepted here. Furthermore, the viscoelastic stretching is not limited.

\section{Constitutive model}

An outgrowth of the imposed symmetry of $\hat{\boldsymbol{F}}^{\mathrm{ve}}$ is that the plastic spin becomes

$$
\hat{\boldsymbol{W}}^{\mathrm{p}}=\operatorname{skew}\left(\dot{\hat{\boldsymbol{F}}}^{\mathrm{p}} \hat{\boldsymbol{F}}^{\mathrm{p}-1}\right)=\dot{\boldsymbol{R}}^{\mathrm{ve}} \boldsymbol{R}^{\mathrm{ve}, \mathrm{T}}+\boldsymbol{R}^{\mathrm{ve}} \overline{\boldsymbol{W}}^{\mathrm{p}} \boldsymbol{R}^{\mathrm{ve}, \mathrm{T}},
$$

i.e. it is nonzero. To define the alignment of the stress-free intermediate placement, the plastic spin is calculated by introducing an algorithmically consistent plastic spin $\tilde{\boldsymbol{W}}^{\mathrm{p}}$, which becomes skew-symmetric finally at the integration interval. A similar scheme is applied in Holopainen (2013); Engqvist et al. (2016). Assuming that the current deformation gradient $\boldsymbol{F}$ can be extracted during incremental calculations, which is the situation in standard finite element codes, the state variables that need to be solved are $\boldsymbol{F}^{\mathrm{ve}}, \tilde{\boldsymbol{W}}^{\mathrm{p}}, \boldsymbol{F}^{\mathrm{v}}$, and $s_{1}$ while $\boldsymbol{F}^{\mathrm{p}}$ and $\boldsymbol{F}^{\mathrm{e}}$ are solved from the decompositions (1) and (6), respectively. To compress the notation, the accent $(\hat{\cdot})$, used for the deformation measures to define the intermediate placements, has been omitted. The variable $s_{1}$ is defined by (66).

Since the model is rate-dependent and long-term periods are investigated, the algorithmic update is conducted by using an implicit (backward Euler) scheme. This method has been reported to be effective for rate-dependent solids, especially under long-term periods, Miehe et al. (1993); Stein and Sagar (2008). To integrate the plastic flow, the exponential update of $\boldsymbol{F}^{\mathrm{p}}$ by Weber and Anand (1990); Miehe et al. (1993) is applied, i.e. the formula

$$
\boldsymbol{F}^{\mathrm{ve}}=\boldsymbol{F} \boldsymbol{F}_{n}^{\mathrm{p}-1} \exp \left(-\Delta t \tilde{\boldsymbol{L}}^{\mathrm{p}}\right)
$$

in which $\tilde{\boldsymbol{L}}^{\mathrm{p}}=\overline{\boldsymbol{D}}^{\mathrm{p}}+\tilde{\boldsymbol{W}}^{\mathrm{p}}$, is used. To flatten the notation, the index $n+1$ for the updated state is neglected, and the quantities at the known state $t_{n}$ are symbolized by $n$.

To evaluate $\tilde{\boldsymbol{W}}^{\mathrm{p}}$ and $\boldsymbol{F}^{\mathrm{v}}$, their symmetry-properties as well as the stress equilibrium (47) are benefited, i.e. the system of nonlinear equations to be solved becomes

$$
\begin{aligned}
\boldsymbol{R}_{1} & :=\boldsymbol{F}^{\mathrm{ve}}-\boldsymbol{F} \boldsymbol{F}_{n}^{\mathrm{p}-1} \exp \left(-\Delta t \tilde{\boldsymbol{L}}^{\mathrm{p}}\right), \\
\boldsymbol{R}_{2} & :=\operatorname{skew}\left(\boldsymbol{F}^{\mathrm{ve}}\right), \\
\boldsymbol{R}_{3} & :=\operatorname{sym}\left(\tilde{\boldsymbol{W}}^{\mathrm{p}}\right), \\
\boldsymbol{R}_{4} & :=\operatorname{skew}\left(\boldsymbol{F}^{\mathrm{v}}\right), \\
\boldsymbol{R}_{5} & :=\ln \boldsymbol{v}^{\mathrm{v}}-\ln \boldsymbol{v}_{n}^{\mathrm{v}}-\Delta t \mathrm{~d}\left(\ln \boldsymbol{v}^{\mathrm{v}}\right) / \mathrm{d} t, \\
R_{6} & :=\left(s_{1}-s_{1, n}-\Delta t \dot{s}_{1}\right) / s_{\mathrm{ss}} .
\end{aligned}
$$

The viscoelastic stretch $\boldsymbol{v}^{\mathrm{v}}$ that appears in (67) is defined by (9). Moreover, the material time derivative of the tensor logarithm $\ln \boldsymbol{v}^{\mathrm{v}}$ is not directly available, 
but is determined by exploiting the corresponding rate of deformation $\boldsymbol{d}^{\mathrm{v}}$ defined by (47), see Hoger (1986) for a detailed account. In this relation, owing to the symmetry of $\boldsymbol{F}^{\mathrm{v}}$, all the rotation terms vanish. The residuals $\boldsymbol{R}_{2}$ and $\boldsymbol{R}_{4}$ consist of only upper (lower) non-diagonal three components and the residuals $\boldsymbol{R}_{3}$ and $\boldsymbol{R}_{5}$ consist of six equations.

Solution of (67) is performed by using Newton-Raphson procedure. When solving the system (67), the state variables are given in the vectorized form, i.e.

$$
\boldsymbol{Y}:=\left[\begin{array}{llll}
\boldsymbol{F}^{\mathrm{ve}} & \tilde{\boldsymbol{W}}^{\mathrm{p}} & \boldsymbol{F}^{\mathrm{v}} & s_{1}
\end{array}\right]
$$

and the solution becomes $\boldsymbol{Y}_{\mathrm{n}+1}=\boldsymbol{Y}_{\mathrm{n}}+\Delta \boldsymbol{Y}$ with the increment

$$
\Delta \boldsymbol{Y}=-\boldsymbol{J}^{-1} \boldsymbol{R}, \text { and the Jacobian } \quad \boldsymbol{J}:=\frac{\partial \boldsymbol{R}}{\partial \boldsymbol{Y}}
$$

The vectorized form $\boldsymbol{R}:=\left[\begin{array}{llllll}\boldsymbol{R}_{1} & \boldsymbol{R}_{2} & \boldsymbol{R}_{3} & \boldsymbol{R}_{4} & \boldsymbol{R}_{5} & R_{6}\end{array}\right]$ consists of the residuals introduced in (67).

\section{Fatigue model}

The differentiation of $\beta$-function (51), required to integrate the evolution equations (53) and (56) for fatigue, also needs to be defined. Assuming that an increment of the strain tensor $\mathrm{d} \boldsymbol{\epsilon}$ is available. Then, based on the relation (13),

$$
\mathrm{d} \boldsymbol{\epsilon}=\frac{1}{2}\left(\mathrm{~d} \boldsymbol{l}+\mathrm{d} \boldsymbol{l}^{\mathrm{T}}\right)
$$

which relation defines the increment $\mathrm{d} \boldsymbol{F}:=\mathrm{d} t \dot{\boldsymbol{F}}$ as

$$
\mathrm{d} \boldsymbol{l}:=\mathrm{d} \boldsymbol{F} \boldsymbol{F}^{-1} .
$$

Using (11), one obtains

$$
\mathrm{d} \boldsymbol{l}:=\mathrm{d} \boldsymbol{l}^{\mathrm{ve}}+\boldsymbol{F}^{\mathrm{ve}} \mathrm{d} \overline{\boldsymbol{L}}^{\mathrm{p}} \boldsymbol{F}^{\mathrm{ve}-1}
$$

where

$$
\mathrm{d} \boldsymbol{l}^{\mathrm{ve}}:=\mathrm{d} \boldsymbol{F}^{\mathrm{ve}} \boldsymbol{F}^{\mathrm{ve}-1} \text { and } \mathrm{d} \overline{\boldsymbol{L}}^{\mathrm{p}}:=\mathrm{d} t\left(\overline{\boldsymbol{D}}^{\mathrm{p}}+\tilde{\boldsymbol{W}}^{\mathrm{p}}\right)
$$

express the incremental counterparts for the viscoelastic and plastic velocity gradients. Due to the symmetry of $\mathrm{d} \boldsymbol{F}^{\mathrm{ve}}$ at the end of the integration interval,

$$
\mathrm{d} \boldsymbol{F}^{\mathrm{ve}}=\frac{1}{2}\left[\left(\mathrm{~d} \boldsymbol{\epsilon}-\boldsymbol{F}^{\mathrm{ve}} \mathrm{d} \overline{\boldsymbol{L}}^{\mathrm{p}} \boldsymbol{F}^{\mathrm{ve}-1}\right) \boldsymbol{F}^{\mathrm{ve}}+\boldsymbol{F}^{\mathrm{ve}, \mathrm{T}}\left(\mathrm{d} \boldsymbol{\epsilon}-\boldsymbol{F}^{\mathrm{ve}-\mathrm{T}} \mathrm{d} \overline{\boldsymbol{L}}^{\mathrm{p}, \mathrm{T}} \boldsymbol{F}^{\mathrm{ve}, \mathrm{T}}\right)\right] .
$$

Substitution of (74) into (73) gives a result which in (72) leads to the formula for $\mathrm{d} \boldsymbol{l}^{\mathrm{ve}}$ and $\mathrm{d} \boldsymbol{l}$, respectively.

The differentiation of $\beta$, defined by (51), results in

$$
\mathrm{d} \beta=\left[\frac{3}{2} \frac{\left(\boldsymbol{\tau}^{\mathrm{dev}}-\boldsymbol{\beta}^{\mathrm{dev}}-\boldsymbol{\alpha}\right)}{\bar{\tau}}: \mathrm{d} \boldsymbol{\tau}^{\mathrm{dev}, \nabla}+a \operatorname{trace}\left(\mathrm{d} \boldsymbol{\tau}^{\nabla}\right)\right] /\left(\sigma_{0}+C \bar{\tau}\right)
$$


where $\mathrm{d} \beta:=\mathrm{d} t \dot{\beta}$ and $\mathrm{d} \tau^{\nabla}:=\mathrm{d} t \tau^{\nabla}$ is an objective stress increment determined later on.

According to (53), in which $\mathrm{d} \boldsymbol{\alpha}^{\nabla}=\mathrm{d} t \boldsymbol{\alpha}^{\nabla}$,

$$
\mathrm{d} \boldsymbol{\beta}^{\nabla}:=\mathrm{d} t \boldsymbol{\beta}^{\nabla}=\boldsymbol{F} \mathrm{d}\left(\boldsymbol{F}^{-1} \boldsymbol{\beta} \boldsymbol{F}^{-\mathrm{T}}\right) \boldsymbol{F}^{\mathrm{T}}=\mathrm{d} \boldsymbol{\beta}-\mathrm{d} \boldsymbol{l} \boldsymbol{\beta}-\boldsymbol{\beta} \mathrm{d} \boldsymbol{l}^{\mathrm{T}}
$$

and

$$
\mathrm{d} \boldsymbol{\beta}:=\boldsymbol{F}^{\mathrm{ve}} \mathrm{d} \overline{\boldsymbol{B}}^{\nabla} \boldsymbol{F}^{\mathrm{ve}, \mathrm{T}}+\mathrm{d} \boldsymbol{l}^{\mathrm{ve}} \boldsymbol{\beta}+\boldsymbol{\beta} \mathrm{d} \boldsymbol{l}^{\mathrm{ve}, \mathrm{T}} .
$$

Differentiation of (61) results in

$$
\frac{\partial \lambda_{\mathrm{ec}}^{\mathrm{p}}}{\partial \overline{\boldsymbol{C}}^{\mathrm{p}}}=\overline{\boldsymbol{I}} /\left(6 \lambda_{\mathrm{ec}}^{\mathrm{p}}\right),
$$

and this result in (62) gives

$\mathrm{d} \overline{\boldsymbol{B}}^{\nabla}=\frac{C_{\mathrm{R}} \sqrt{N}}{3 \lambda_{\mathrm{ec}}^{\mathrm{p}}}\left[\left(\frac{\chi_{\mathrm{ec}} \xi_{\mathrm{ec}}}{3 \sqrt{N} \lambda_{\mathrm{ec}}^{\mathrm{p}}}-\frac{1}{3\left(\lambda_{\mathrm{ec}}^{\mathrm{p}}\right)^{2}}\right) \operatorname{trace}\left(\mathrm{d} \overline{\boldsymbol{L}}^{\mathrm{p}} \overline{\boldsymbol{C}}^{\mathrm{p}}\right) \overline{\boldsymbol{C}}^{\mathrm{p}}+2 \operatorname{sym}\left(\mathrm{d} \overline{\boldsymbol{L}}^{\mathrm{p}} \overline{\boldsymbol{C}}^{\mathrm{p}}\right)\right] \mathfrak{L}^{-1}\left(\frac{\lambda_{\mathrm{ec}}^{\mathrm{p}}}{\sqrt{N}}\right)$

wherein $\chi_{\mathrm{ec}}:=1 / \mathfrak{L}^{-1}\left(\lambda_{\mathrm{ec}}^{\mathrm{p}} / \sqrt{N}\right)$. The inverse Langevin function $\mathfrak{L}^{-1}$ is calculated using the Padé-approximation, see Steinmann and Stein (1996).

Lastly, the stress increment needed in (75) becomes

$$
\mathrm{d} \boldsymbol{\tau}^{\nabla}=\boldsymbol{F} \mathrm{d}\left(\boldsymbol{F}^{-1} \boldsymbol{\tau} \boldsymbol{F}^{-\mathrm{T}}\right) \boldsymbol{F}^{\mathrm{T}}=\mathrm{d} \boldsymbol{\tau}-\boldsymbol{l} \boldsymbol{\tau}-\boldsymbol{\tau} \boldsymbol{l}^{\mathrm{T}}=\mathfrak{c}: \mathrm{d} \boldsymbol{\epsilon}
$$

where

$$
\mathfrak{c}:=\frac{\mathrm{d} \boldsymbol{\tau}}{\mathrm{d} \boldsymbol{F}}:(\boldsymbol{i} \odot \boldsymbol{F})-\boldsymbol{i} \odot \boldsymbol{\tau}-\boldsymbol{\tau} \odot \boldsymbol{i}
$$

is the algorithmic tangent stiffness (ATS) tensor defined using a non-standard tensor product ${ }^{4}$

$$
(\boldsymbol{A} \odot \boldsymbol{B})_{i j k l}=1 / 2\left(\boldsymbol{A}_{i l} \boldsymbol{B}_{j k}+\boldsymbol{A}_{i k} \boldsymbol{B}_{j l}\right) .
$$

In view of (47),

$$
\frac{\mathrm{d} \boldsymbol{\tau}}{\mathrm{d} \boldsymbol{F}}=\mathfrak{L}^{\mathrm{e}}: \frac{\partial\left(\ln \boldsymbol{v}^{\mathrm{e}}\right)}{\partial \boldsymbol{v}^{\mathrm{e}}}: \frac{\mathrm{d} \boldsymbol{v}^{\mathrm{e}}}{\mathrm{d} \boldsymbol{F}}
$$

wherein the differentiation of the tensor logarithm is considered following Miehe et al. (1993).

Based on the fact that the residuals $\boldsymbol{R}$ in (67) vanish finally at the integration interval for all $\boldsymbol{F}$, i.e. $\boldsymbol{R}(\boldsymbol{Y}(\boldsymbol{F}), \boldsymbol{F})=\mathbf{0}$, one obtains

$$
\frac{\mathrm{d} \boldsymbol{Y}}{\mathrm{d} \boldsymbol{F}}=-\left(\frac{\partial \boldsymbol{R}}{\partial \boldsymbol{Y}}\right)^{-1} \frac{\partial \boldsymbol{R}}{\partial \boldsymbol{F}}=:-\boldsymbol{J}^{-1} \frac{\partial \boldsymbol{R}}{\partial \boldsymbol{F}} .
$$

\footnotetext{
${ }^{4}$ The component representation of this tensor product is general and does not include all possible (sub)symmetries needed in its numerical development. Treatment of possible symmetries of high order tensors including different tensor products is discussed in (Holopainen, 2014, Sec. 6).
} 
Using (83), one can also extract $\mathrm{d} \boldsymbol{v}^{\mathrm{e}} / \mathrm{d} \boldsymbol{F}$ that is consistent with the applied integration method and thus, all components for the calculation of the ATStensor (81) are available.

In different finite-element softwares, the objective stress rate and increment needed in the equilibrium iterations can differ from (80). One generally used measure is the Jaumann rate, $\boldsymbol{\sigma}^{\nabla J}$, Stein and Sagar (2008). Based on (80), its incremental representation is defined by

$$
\mathrm{d} \boldsymbol{\sigma}^{\nabla \mathrm{J}}:=\mathrm{d} t \boldsymbol{\sigma}^{\nabla \mathrm{J}}=J^{\mathrm{ve}-1} \mathrm{~d} \boldsymbol{\tau}^{\nabla}+\mathrm{d} \boldsymbol{\epsilon} \boldsymbol{\sigma} \boldsymbol{\sigma} \mathrm{d} \boldsymbol{\epsilon}-\operatorname{trace}(\mathrm{d} \boldsymbol{\epsilon}) \boldsymbol{\sigma}=\boldsymbol{c}^{\sigma \mathrm{J}}: \mathrm{d} \boldsymbol{\epsilon}
$$

where

$$
\mathfrak{c}^{\sigma \mathrm{J}}=J^{\mathrm{ve}-1} \mathfrak{c}+\boldsymbol{i} \odot \boldsymbol{\sigma}+\boldsymbol{\sigma} \odot \boldsymbol{i}-\boldsymbol{\sigma} \otimes \boldsymbol{i} .
$$

An implicit Euler scheme is also used for updating unknown fatigue variables. Integration of the internal rate variables (53) and (56) results in the nonlinear residual functions for fatigue, i.e.

$$
\begin{aligned}
\boldsymbol{R}_{f, 1} & :=\boldsymbol{\alpha}-\boldsymbol{\alpha}_{\mathrm{n}}-\mathrm{d} t \boldsymbol{\alpha}^{\nabla}, \\
R_{f, 2} & :=D-D_{\mathrm{n}}-\mathrm{d} t \dot{D}
\end{aligned}
$$

where $\mathrm{n}$ refers to the quantities from the last state of equilibrium.

The proposed algorithm for a solution of the systems (67) and (85) is based on the assumption that those two systems are considered uncoupled, i.e. they are solved in succession, and the fatigue damage does not influence the global tangent stiffness given by (81). This assumption, as discussed in Introduction, is based on the experimental observations that fatigue damage represents solely the formation of micro-cracks that has not a marked influence on macroscopic stresses and deformations. Moreover, the formation of micro-cracks and initial crazing typically cover most of (over $90 \%$ ) the total fatigue life of amorphous polymers, Janssen et al. (2008).

\section{Model evaluation}

\subsection{Calibration of the model}

The model parameters were determined from cold drawing experiments on injection-molded dogbone-shaped PC-specimens (Lexan $\left.{ }^{\circledR} 223 \mathrm{R}\right)$. The test method and specimen geometry are defined by ASTM D638-IV, Foster (2015). The specimen is also termed a uniaxial tensile bar since the stress state in the specimen's web is virtually uniaxial. During loadings, the elongation $u$ against the applied force $f$ was monitored. Due to the inhomogeneous deformations of the specimen, the tests were simulated and therefore, the model was implemented in a finite-element program with the mesh consisting of linear solid elements.

The elastic Young's modulus $E$ was first determined from the initial linear region of the $f-u$ curve. Value of the Poisson's ratio was taken from Dreistadt et al. (2009). The plasticity model parameters were found from the least squares fitting to monotonic tension experiments with the presence of 

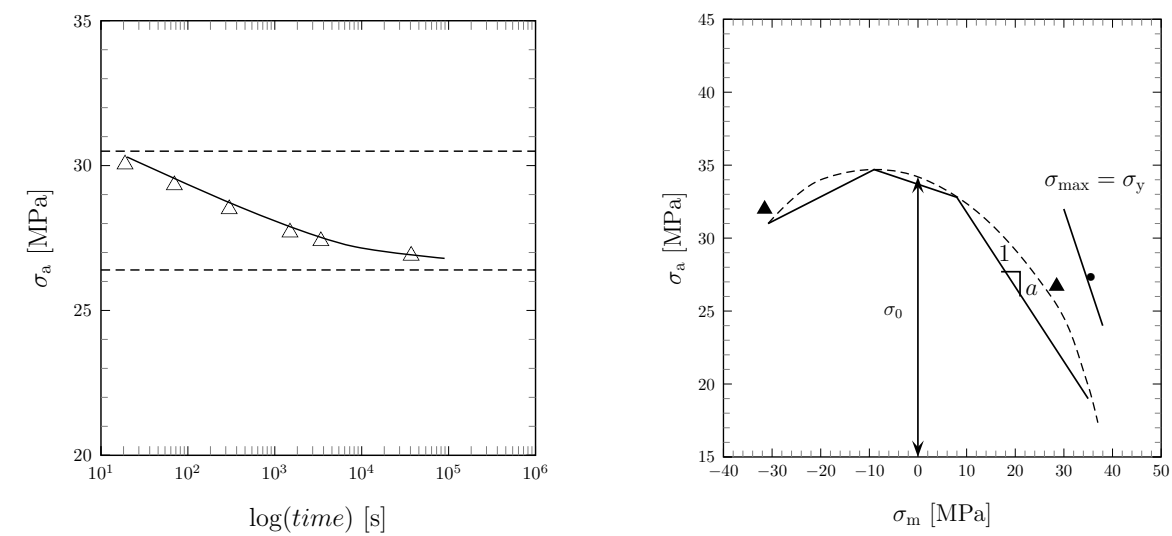

Figure 4: Fatigue amplitudes of a PC polymer subjected to accelerated tests with the mean stress of $\sigma_{\mathrm{m}}=\sigma_{\mathrm{a}}+2.2 \mathrm{MPa}$ (left). The thick line implies the model response, and the symbol $\boldsymbol{\Delta}$ denotes the data points extracted from Janssen et al. (2008). The two dashed lines demonstrate the static tensile yield strength $\sigma_{\mathrm{y}}$ (upper) and an approximated endurance limit (lower). The endurance limit at different mean stresses, i.e. the Haigh-diagram (solid line) according to the model (right). The material parameter $a$ assigns the slope, and the dashed curve means the supposed experimental response. The tangent line at the ultimate stress point $\sigma_{\max }=\sigma_{\mathrm{y}}$ at $\sigma_{m}=35 \mathrm{MPa}$ is also shown.

strain softening and subsequent hardening in large strains. The remaining parameters for viscoelasticity $\left(E^{\mathrm{v}}, \eta\right)$ were extracted from the tension tests for creep and recovery. The tests encompassed serial unloadings to not equal stress levels followed by different dwell periods at a constant stress levels. Details of the experiment can be found in the study by Holopainen (2013). The model parameters are given in Table 1.

Once finding of the constitutive model parameters, the fatigue model parameters were extracted from in situ measurements taken from Janssen et al. (2008) (Lexan ${ }^{\circledR} 101 \mathrm{R}$ and 161R). Isothermal test conditions at room temperature included a uniaxial stress submitted to load control and measured at the crosshead of the applied uniaxial test specimens. Since the formation of microcracks governs the majority (up to more than 95\%) of the entire fatigue life, Marissen et al. (2001); Janssen et al. (2008), fatigue damage solely under this period is investigated.

An accelerated life experiment based on a high mean stress has been followed, Maxwell et al. (2005); Janssen et al. (2008); Kim and Lu (2008). In accordance with the experiments conducted by Janssen et al. (2008), the applied waveform is sinusoidal, and the maximum stress is enabled to alter, whereas the minimum stress is kept at a constant of $2.2 \mathrm{MPa}$. Isothermal testing conditions are guaranteed by using a low frequency of $2 \mathrm{~Hz}$. The procedure for the calibration of the fatigue parameters is discussed in more detail in (Holopainen et al., 2017, Section 3.2). The calibrated model parameters and responses are shown in Table 2 and Fig. 4, respectively. 


\subsection{Validation - uniaxial stress}

Considering first the static creep loadings $\left(\sigma=\sigma_{\mathrm{m}}\right)$, cf. Fig. 5(left). Aging kinetics has no importance in lifetimes in this stress regime at room temperature, where the experimental results suggest virtually a linear response. The model pursues to overestimate life spans, which feature is owing to an increase in the endurance surface at mean stresses far grater than the ones used in the calibration. Obviously, the model result could be improved by exploiting a larger set of data in calibration, cf. Engqvist et al. (2016).

Anand and Ames (2006) conducted cyclic compression tests on polymethyl methacrylate (PMMA) wherein the specimen was first pre-strained up until the logarithmic strain level of $20 \%$ was reached and then cyclically strained between this and zero strain. The proposed model was also calibrated to this test, and Fig. 5 shows that the model without the viscoelastic constitutive treatment cannot predict well the experimental response. Due to the proposed viscoelastic model, the backstress smoothly varies during the loading loops, when the model is able to predict a nonlinear response during both loading and unloading and, in contrast to a purely elastic deformation, a suitable loss of mechanical energy, equal to the area of experimentally observed hysteresis loops. After few cycles, the model response stabilizes and shows a saturated state of hardening similar to the experimental response, Anand and Ames (2006); Holopainen et al. (2017).

Fig. 6 also shows that an exaggerated creep is predicted by the model without viscoelasticity. However, neither the original nor the extended model can reproduce the stress peaks during reloading but tend directly towards the monotonic loading path, cf. Dreistadt et al. (2009). This behavior refers to an unexpected isotropic hardening, i.e. the model should be enhanced for this part.

Owing to the linear relationship between the effective stress and the hydrostatic stress in the expression of the endurance surface (51), the model cannot predict fatigue under a notable negative mean stress using a single value of the slope parameter $a$, see Fig. 4. Probably the simplest modification is to use dif-

Table 1: Constitutive model parameters for a PC polymer fitted to cold drawing experiments. The rest of the parameters for the viscoelastic model are $E^{\mathrm{e}}:=E=2300$ $\mathrm{MPa}, E^{\mathrm{v}}=1050 \mathrm{MPa}, \eta=0.5 \cdot 10^{5} \mathrm{MPas}$, and $\nu^{\mathrm{e}}=\nu^{\mathrm{v}}:=\nu=0.37$.

\begin{tabular}{lcccccccc}
\hline \hline Parameter & $s_{0}$ & $s_{\mathrm{ss}}$ & $h_{1}$ & $\dot{\gamma}_{0}$ & $C^{\mathrm{R}}$ & $N$ & $A$ & $\alpha$ \\
\hline Unit $\ldots \ldots \ldots$ & $\mathrm{MPa}$ & $\mathrm{MPa}$ & $\mathrm{MPa}$ & $1 / s$ & $\mathrm{MPa}$ & & $\mathrm{K} / \mathrm{MPa}$ & \\
Value $\ldots \ldots \ldots . .$. & 97 & 77 & 715 & $5.6 \cdot 10^{15}$ & 14 & 2.2 & 240 & 0.08 \\
\hline \hline
\end{tabular}

Table 2: Parameters of the fatigue model for a PC polymer.

\begin{tabular}{lccccccc}
\hline \hline Parameter & $\sigma_{0}$ & $a$ & $C$ & $K \cdot 10^{6}$ & $L_{1}$ & $L_{2}$ & $\vartheta$ \\
\hline Unit ......... & $\mathrm{MPa}$ & & & & & & \\
Value ........ & 33.8 & 0.46 & 0.05 & 8.6 & 7.7 & 3.8 & 2.65 \\
\hline \hline
\end{tabular}



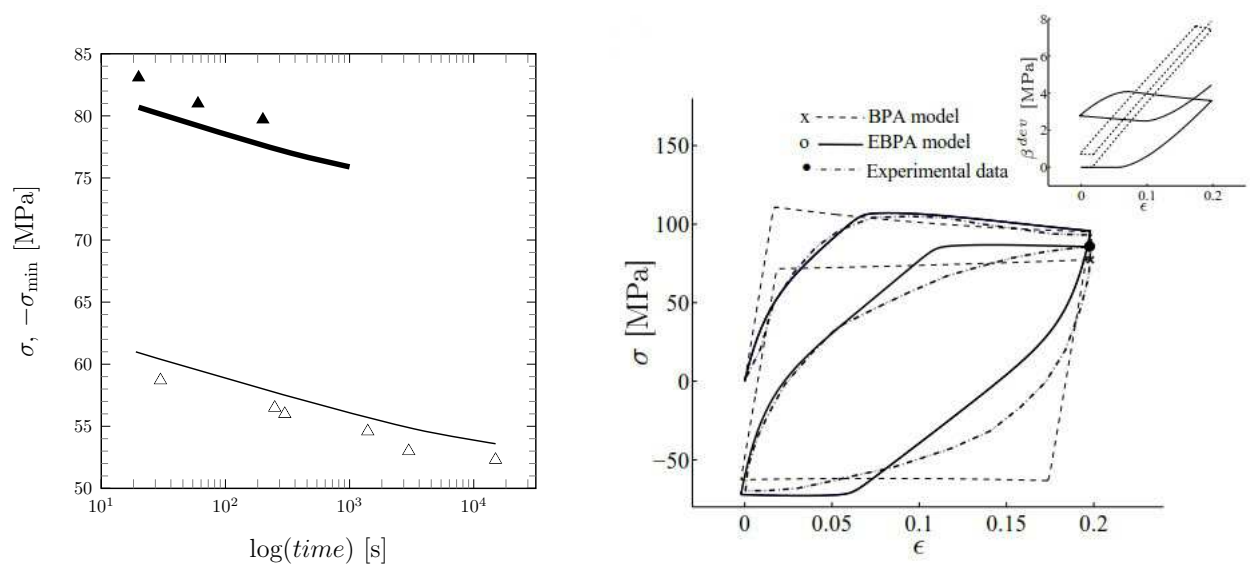

Figure 5: The lifetime underneath static creep loading, $\sigma_{\mathrm{a}}=0$ (left). The thin line and the markers $\triangle$ are the model response and data points taken from Janssen et al. (2008). Lifetimes under a uniaxial compression when $\sigma_{\max }=2.2 \mathrm{MPa}$ is also shown. The model response and data points taken from Janssen et al. (2008) are signified by the thick solid curve and black symbols. Comparison of the proposed (extended Boyce-Park-Argon model, EBPA-model) and the original (BPA) model curves against the experimental data for PMMA (right). Experimental data is taken from Anand and Ames (2006) and the model responses are calculated using the calibrated parameters given in Holopainen (2013).

ferent values for high compression, high tension, and their intermediate region, Fig. 4. Here, a negative value $a=-0.17$ is used for the high compression applied. In spite of calibration being applied solely for high tension, this proposed elementary upgrade results in a model response that complies rather well the available data points under a high compression, cf. Fig. 5(left). Both the model and experimental results indicate that the fatigue strengths under tension are significantly lower than that under compression.

The given approach also produces time-to-failure predictions with reasonable accuracy under different stress ratios $R$ (the ratio between the smallest and largest stresses). The model predicts the data for the low stress ratio $R=0.1$ rather well, but underestimates a little the fatigue lives at higher stress ratios, see Fig. 7. The difference stems from the calibration being applied solely to low stress ratio loadings as well as to a slightly different PC applied in the experiments. It appears that both the model and experimental response point out an asymptotic demeanor at the extremes of the lifetime and virtually equal endurance limits, cf. (Kanters et al., 2016, Fig. 8a). Fig. 7 also demonstrates the idea of the approach. It emerges from data that the service life is practically governed by a ductile fatigue damage development prior to a rapid damage growth caused by crack propagation that ultimately leads to the component's brittle failure.

Fig. 7 further shows a fatigue life plot and an endurance limit under fully reversed strain-controlled loadings (no ratcheting). In this strain regime, hysteresis loops possess a minimal hysteresis loop area, see (Hughes et al., 2017, Fig. 6). Data for the applied PC-polymer indicates a low endurance limit. In 

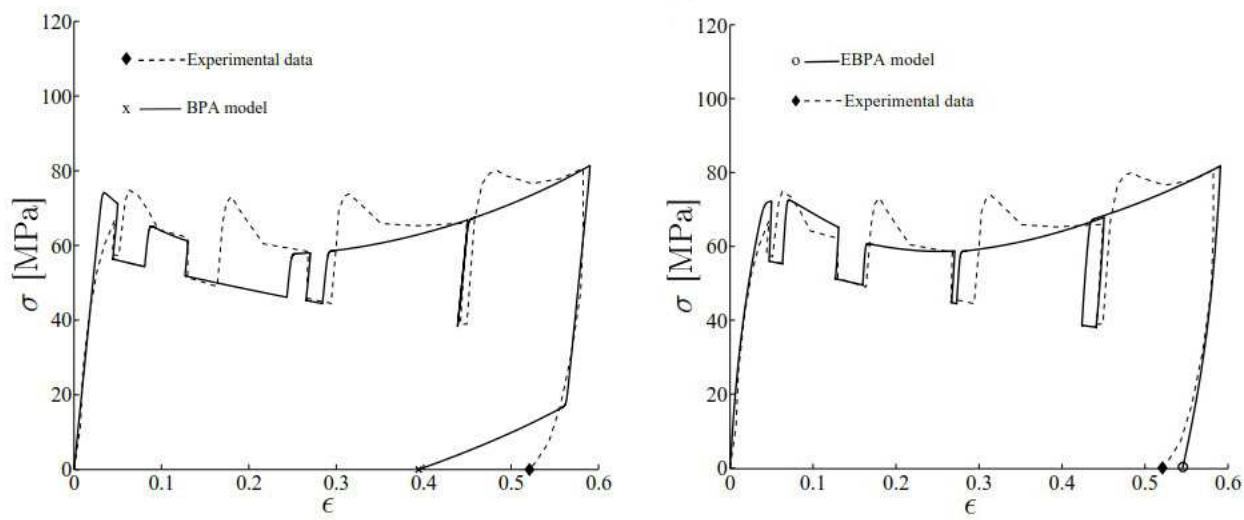

Figure 6: Uniaxial compression responses for Bisphenol A (BPA) polymer according to the BPA and the EBPA model. The repeated unloadings are performed to $59 \mathrm{MPa}$ (in terms of the first Piola-Kirchhoff stress) and then kept at this level for 12,000 seconds. Experimental data is taken from Dreistadt et al. (2009). Responses for PBA are calculated using the calibrated parameters given in Holopainen (2013).
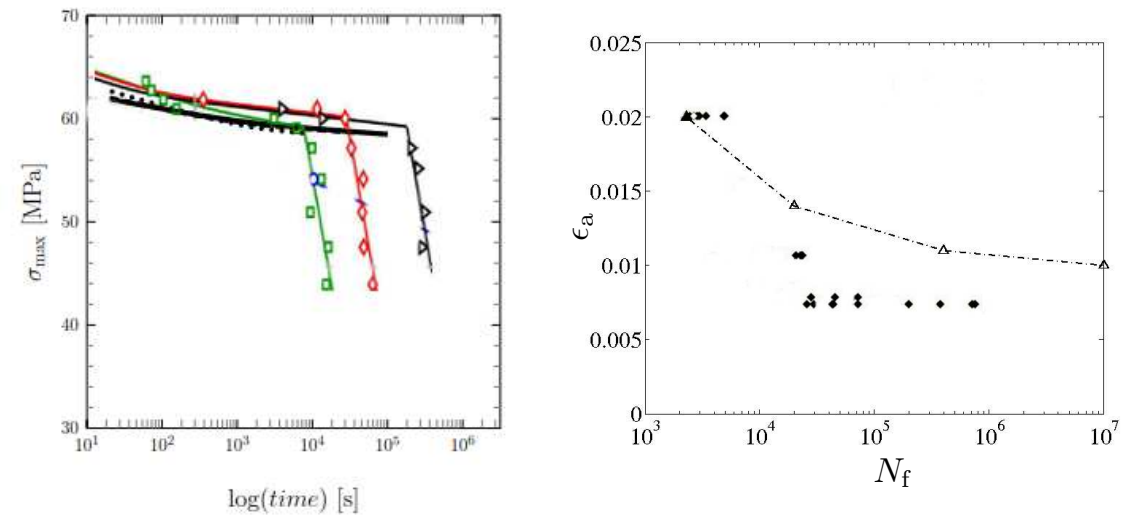

Figure 7: Time to failure of PC (Lexan ${ }^{\circledR} 143 \mathrm{R}$ ) for $R$-values of $0.1,0.4$, and 0.7 (left). Black dotted curve, and black dashed and solid curves (overlapping) represent the model results, respectively, while the green, red, and black curves with markers are the corresponding data points extracted from Kanters et al. (2016). The waveform is sinusoidal with a frequency $1 \mathrm{~Hz}$. Predicted and experimental (markers $\downarrow$, taken from Hughes et al. (2017)) strain amplitude vs fatigue life plots under fully reversed uniaxial strain-controlled loadings with the frequency of $10 \mathrm{~Hz}$ (right).

calculations, the strength limit $\sigma_{0}=27 \mathrm{MPa}$ and the reduced slope $a=0.2$ have been applied, see Fig. 4. Before the endurance limit and a plateau at lower strain amplitudes, a quasi-linear section can be observed both in data and the model response. The difference between the data and model response is first due to the calibration being applied solely to tension loadings and secondly due to a slightly different PC applied in the experiments causing a temperature increase under the applied load frequency.

The endurance surface (51) predicted under a cyclic tension is illustrated in Fig. 8. The endurance surface rapidly tracks the periodic stress state since the combined backstress $\boldsymbol{\alpha}+\boldsymbol{\beta}^{\mathrm{dev}}$ invariably moves in the direction of $\boldsymbol{\tau}^{\mathrm{dev}}-\boldsymbol{\beta}^{\mathrm{dev}}-\boldsymbol{\alpha}$ 

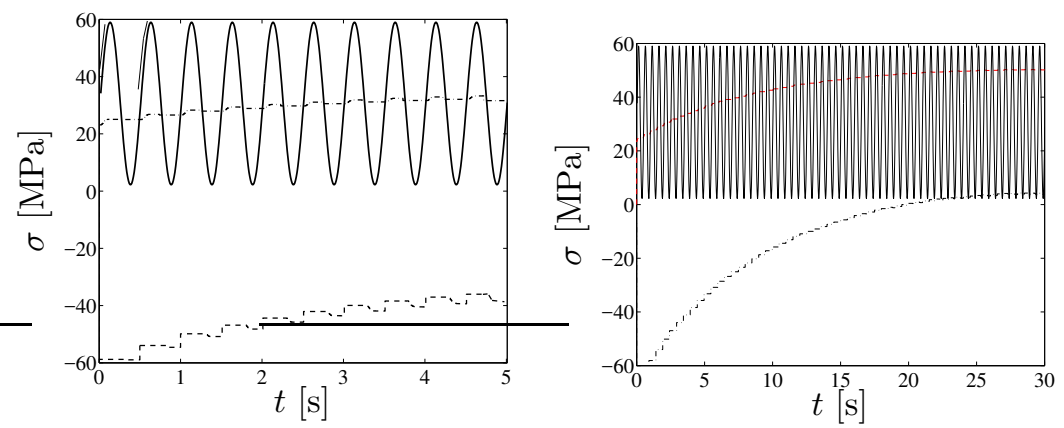

Figure 8: The stress (solid line) and the development of the endurance surface (nonsolid lines) during the first few cycles (left). Development of damage during the first cycles is highlighted by the double curve. The corresponding responses when a periodic state of the endurance surface has been reached (right).
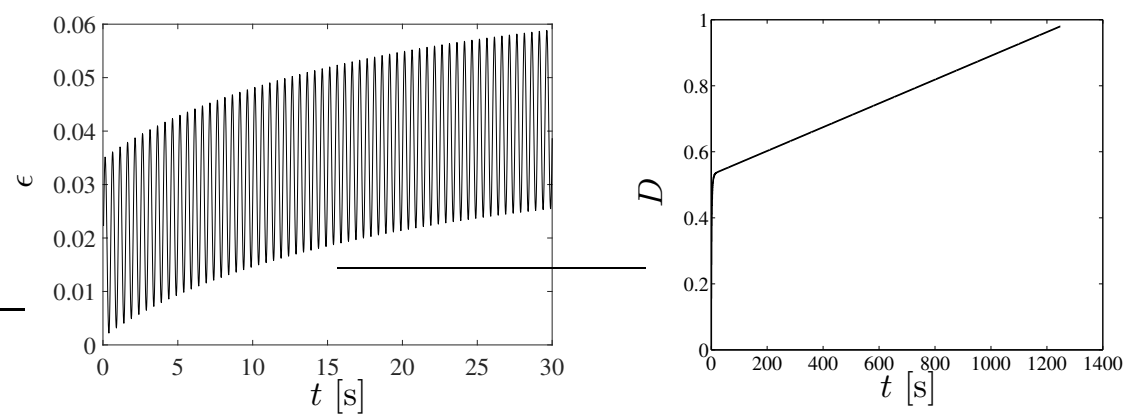

Figure 9: Strain development under the cyclic tension loading shown in Fig. 8 (left). Development of damage variable according to (56) (right).

according to the equation (53) and the plots in Fig. 3. Also, according to Proposition 2.4, damage only grows as the upper endurance limit in Fig. 8 shows an increase and the stress is greater than this limit, i.e. the stress state recedes from the endurance surface. Compared to elastic metals, see Ottosen et al. (2008), reaching of the periodic state requires more cycles, which characteristic is due to ratcheting phenomenon under the applied stress-controlled loading, Lu et al. (2016); Holopainen et al. (2017)

Figure 9 further indicates a periodic development of the logarithmic strain that shows ratcheting or cyclic creep, i.e. strain constantly increases as the fluctuating loading advances, cf. Lemaitre and Desmorat (2005); Lu et al. (2016); Holopainen et al. (2017). A strong initial growth of damage at this phase is owing to the high mean stress applied, cf. Fig. 9(right). During continued loading, an increase in the viscoelastic and plastic strains, especially the latter, leads to a significant elongation of the tensile test specimen that can induce a plastic instability termed necking, which is ultimately followed by a brittle rupture, as depicted in Janssen et al. (2008). Section 3.3 returns to this topic. 

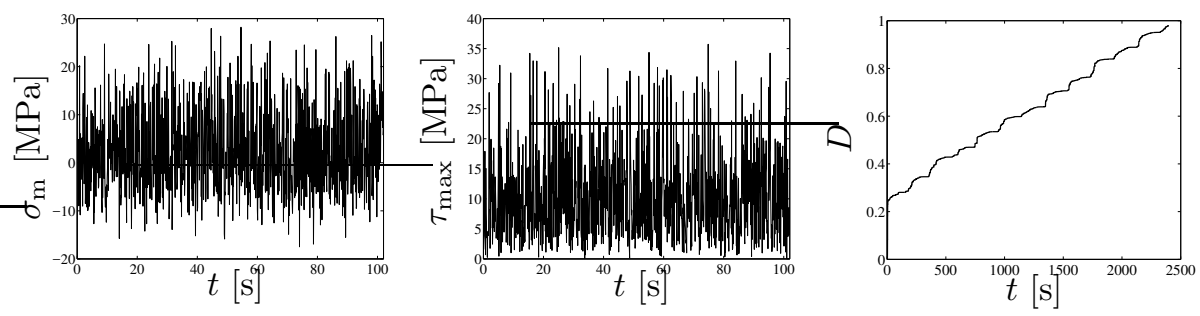

Figure 10: Clip of the mean stress and the maximum shear stress history of a 3D-random loading. Based on the parameters given in Table 2 fatigue damage development up to failure.

\subsection{Multiaxial stresses}

\section{Complex loading}

It is interesting to investigate three dimensional complex cyclic loads in order to take advantage of the incremental formulation of the proposed approach. The time history for all six stress components is randomly generated assuming the stresses vary between the limits $-34 \mathrm{MPa}$ and $58 \mathrm{MPa}$, i.e. the stresses remain below the yield peak stress (over $60 \mathrm{MPa}$ ), which can also be concluded from the clip of the maximum shear stress history shown in Fig. 10. In accordance with the calibration for fatigue, the approach was implemented in a Fortran program using the Euler backward integration scheme. Computations were performed using an Intel Haswell processor running at $2.6 \mathrm{GHz}$. The advantage of adaptive concepts cannot be benefited under fatigue loadings, and a constant time step of $0.1 \mathrm{~s}$ was applied to capture the damage evolution under the given complex load spectrum sufficiently accurately.

Due to the small time step employed, typically only two Newtons corrector iterations per time step were needed to converge within the absolute tolerance of $10 \mathrm{E}-8$ for the Euclidean norm of the residual vector ${ }^{5}$. The computation up to the final failure $(D=1)$ took about $20 \mathrm{~s}$ of CPU time. A significant interest is that the proposed evolution equation-based fatigue model readily results in the damage development under multiaxial complex loading without the need of cycle counting techniques frequently applied in fatigue analyses, see Fig. 10. Computations confirm the expected fatigue damage development and verify the model's capability under multiaxial loading conditions.

\footnotetext{
${ }^{5}$ Considering monotonic loading for strain softening and subsequent hardening in large strains, 2 - 4 Newton's iterations were needed to satisfy the tolerance.
} 
Fatigue of a test specimen

Fatigue in the entire test specimen is investigated using a finite-element method. The same finite-element mesh and test specimen geometry as used in the model calibration are applied, see Section 3.1. The displacement at the specimen's top, $y=0$, is secured by a bracket while the signal for uniformly distributed sinusoidal load at $y=L$ ( $L$ is the length of the specimen) is allowed to vary between $28.0 \mathrm{MPa}$ and $1.1 \mathrm{MPa}$ at the frequency of $2 \mathrm{~Hz}$. The undeformed area of the specimen within the clamps is twofold of that of the web. Thus, in accordance with the applied effective stresses (not reduced by damage), the stress state in the specimen's web is virtually twofold.

The finite element computations were performed based on the Jaumann rate given by (84). A constant time step value $0.015 \mathrm{~s}$ was applied to capture the sinusoidal fatigue loading sufficiently accurately. The number of global iterations have been extracted from a finite element package based on a direct linear equation solver and its default values of tolerances, Abaqus (2012). For instance, the tolerance for residual force has been 5E-3. Owing to the small time step employed, typically only two local Newtons corrector iterations per a global iteration were needed in the integration points to converge within the absolute tolerance of 1E-8 for the Euclidean norm of the local residual vector, see Table 3 .

Since a real material suffers from imperfections or inhomogeneities, experiments show final failure outside the specimen's middle, which is expected to be the most stressed location due to the reduction in the cross section (necking), cf. Janssen et al. (2008). To simulate this characteristic, a weakened narrow section was included and it was modeled by increasing the Young's modulus (10 $\%)$ and reducing the fatigue parameters $K$ and $L_{1}(4 \%)$ in other parts of the specimen. This section widens with elongation of the specimen as shown in Fig. 11.

Since damage does not affect stresses, the stress field in the section and anywhere else in the web is homogeneous. Thus, a single point shown in Fig. 11 (right) is representative for the entire specimen and its stress field is usable for the evaluation of fatigue life. Moreover, it appeared that the axial (longitudinal) stress component in the specimen's web was orders of magnitude larger com-

Table 3: Global iterations per step and local Newton iterations per a single global iteration. Direct sparse method (by Abaqus software) has been used as a solver for the global system of equations, Abaqus (2012). The local iterations have been calculated in a single integration point (using the user material subroutine), closest to the specimen's middle.

\begin{tabular}{lccccccc}
\hline \hline Step No. & $1-4$ & $5-11$ & 12 & $1173-1176$ & $1177-1182$ & $3311-3316$ & $3317-3334$ \\
\hline Global ....... & 3 & 2 & 2 & 2 & 3 & 3 & 2 \\
Local ........ & $1-2$ & $1-2$ & 2 & $1-2$ & $1-2$ & $1-2$ & $1-2$ \\
\hline \hline
\end{tabular}



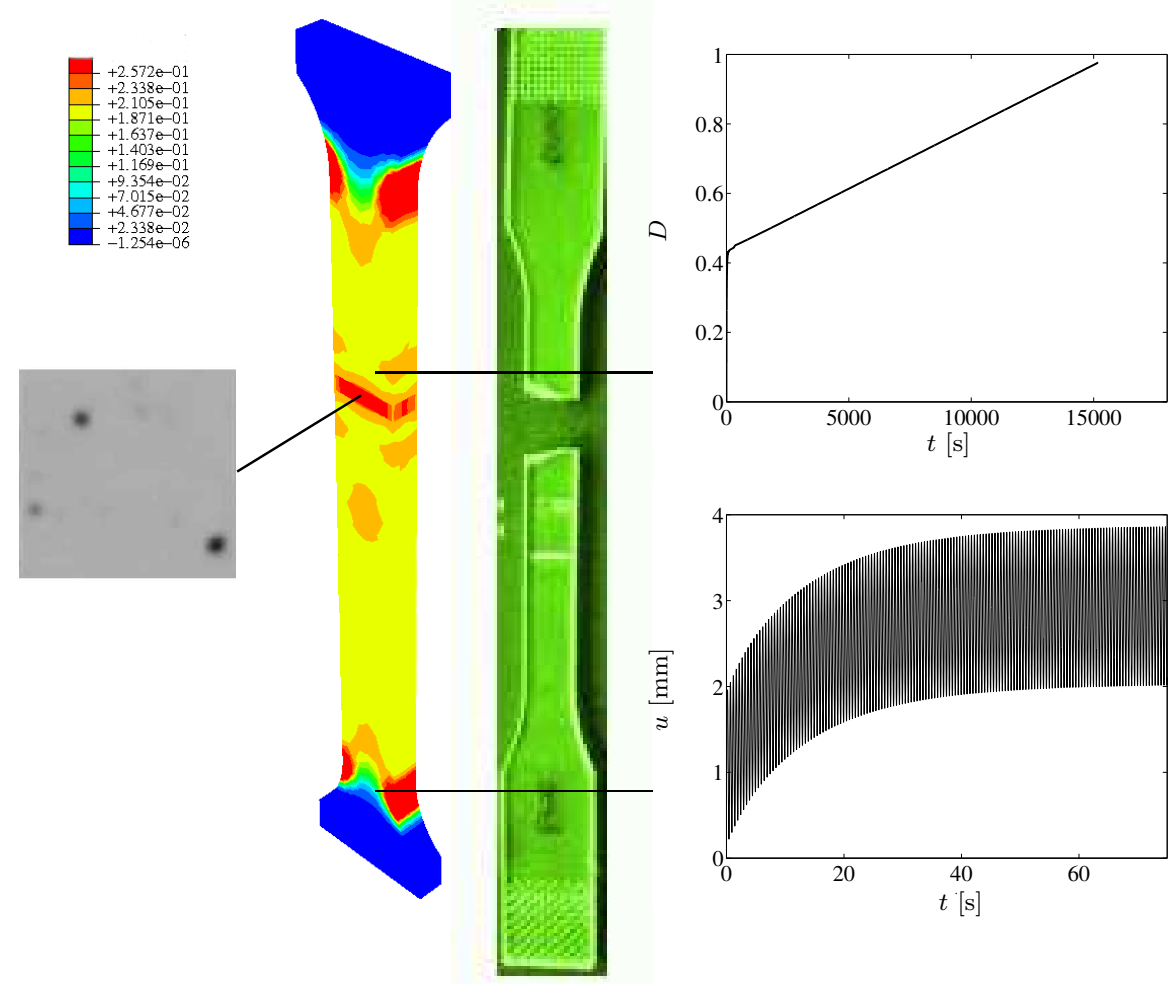

Figure 11: Damage distribution in a tension member instantly after 100 cycles (left). Maximum damage evolution in the specimen's web and elongation $u$ in the specimen's middle (right). The overall lateral strain components in the web are about $2 \nu u / L$ where $L$ is the length of the specimen. Distribution of assumed impurities (black color) in the matrix material is shown in the grey inset.

pared to others, i.e., as was presumed, uniaxial stress state is accurate enough for calibration. Due to this reason, prediction of fatigue life based on the threedimensional stress state (in a single point) shown in Fig. 11 corresponds well to that demonstrated in Fig. 4 under uniaxial loadings.

Although not shown, damaged fields closely resemble the fields of increasing localized plastic deformation and imply that the plastic deformation is a precursor for providing crazing initiation sites that then control propagation of fatigue, cf. Li et al. (1995); Murakami (2012); Lugo et al. (2014). During continued cyclic loading, microscopic cracks extend to wide cracks, which cause component's ultimate failure, Lemaitre and Desmorat (2005); Ding et al. (2017); Lawrimore et al. (2016). Depending on the intensity of loading, the specimen exhibits an instantaneous rupture after necking (low-cycle fatigue) or a brittle fracture after at least several of thousands of cycles, (Janssen et al., 2008, Fig. 4). The fatigue life and attenuated elongation of the specimen without a clear necking implicate the latter case, see Fig. 11. The predicted elongation refers to 

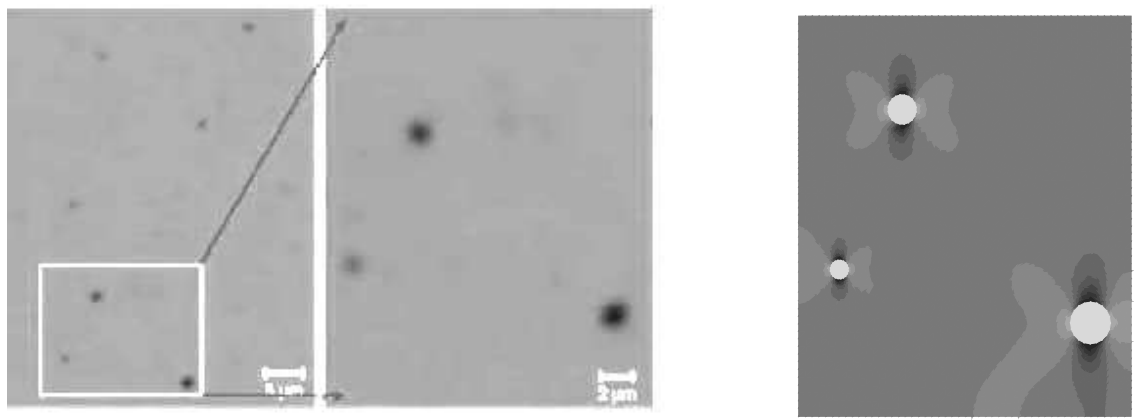

Figure 12: Micrographs of assumed inhomogeneities, cf. Lugo et al. (2014) for ABS polymer (reused with permission from John Wiley and Sons license) (left). The presence of inhomogeneities is demonstrated by the black color. Predicted damage distribution instantaneously after 100 cycles (right). The maximum value of 0.18 is highlighted by the black color. Inhomogeneities were considered linear elastic (Young's modulus was $75 \%$ of the PC-matrix).

one kind of ratcheting, i.e. the elongation $u$ (up to $10 \%$ ) shows a stabilized increase concurrently with the progress of the recurring load, cf. Lu et al. (2016); Holopainen et al. (2017).

\section{Fatigue in RVE}

Fatigue damage in amorphous polymers is closely interconnected to the microstructure and is typically owing to the nucleation of micro-defects or inhomogeneities within a disordered microstructure, thus producing sites for stress intensification, Marissen et al. (2001); Lesser (2002). A distribution of such inhomogeneities is shown in Figs 11 and 12. Once a repeated loading is applied, the said defects can grow and deform and affect fatigue at stress levels far below the nominal yield stress, Marissen et al. (2001); Lesser (2002); Lugo et al. (2014); Kanters et al. (2016).

A microscopic rectangular region representing a representative volume element (RVE) in the most damaged zone of the specimen's web was investigated, see Fig. 11 and 12. The finite-element simulations were conducted based on plane strain assumption (thick element), and the mesh consisted of linear elements. Due to the micro-scale, cyclic loadings of the test specimen in this minute region were considered far-field loadings while the boundaries of the region were considered straight, cf. Steenbrink and Van der Giessen (1998), i.e. displacement-control was applied. The sinusoidal waveforms applied along the top region and the right lateral side were taken from the analysis of the entire specimen, see Fig. 11. Interest was focused on the PC -matrix, i.e. fatigue of inhomogeneities was not taken into consideration in the subsequent study.

The result of the finite-element simulation in Fig. 12 demonstrates that damage starts with the formation of small zones around the inhomogeneities 

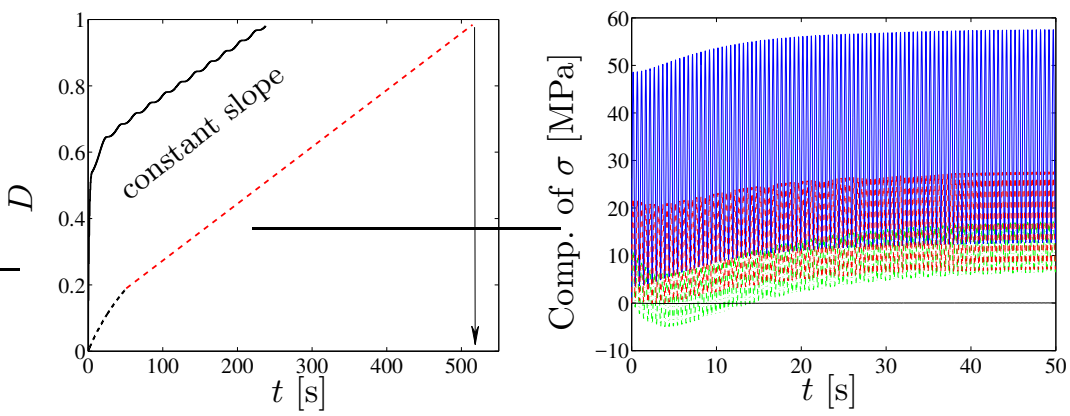

Figure 13: Maximum damage development in a single point of RVE (black solid line) using the stabilized stress components of the last cycle shown right (left). The black dashed curve represents the maximum damage development in RVE based on finiteelement simulation, and the red dashed line with a constant slope is used to estimate the fatigue life, about 1000 cycles. The local stresses, $\sigma_{11}$ (green dash-and-dot line), $\sigma_{12}$ (black solid line), $\sigma_{22}$ (blue solid line, longitudinal), and $\sigma_{33}$ (dashed red line) (right).

and develops rapidly therein while the rest of the material appears not as much damaged and is virtually homogeneous. This result favors the assumption that the damage is highly localized and thus does not have a marked effect on the macroscopic structural response.

Since the stress field is not affected by fatigue damage, it is possible to evaluate the fatigue life by exploiting the initial, three-dimensional stress spectrum observed in a single, most damaged point at an inclusion's top, see Fig. 12. The most representative cycle is the one observed at the end of the simulation when the stress state had stabilized, see Fig. 13. The applied stress field results in a build-up of notable pressure, and this triaxiality promotes local damage development and significantly reduces the fatigue lifetime with respect to that observed in the test, see Figs. 13 and 11, respectively. However, since this damage development represents a single material point, it has no impact on the fatigue life of the entire test specimen.

\section{Concluding remarks}

The article introduces an approach suitable for predicting isothermal fatigue intrinsic to amorphous solids and discusses experimental observations through theory. To ensure practical importance, the proposed approach, based on evolution equations, requires a single set of material parameters and is suited for numerical implementations. The fatigue damage evolution is formulated by exploiting an endurance surface that moves in an effective, damage-free stress space, thus avoiding a complex relationship between the deformation and damage as well as challenges in numerical implementation. The idea is suitable for amorphous solids in which the fatigue behavior is ductile, i.e. damage during the formation of micro-cracks, which inevitably governs the bulk of the entire fa- 
tigue life (over 90\%), is highly localized and thus does not affect the macroscopic stress.

Thermodynamic and numerical treatments of the approach have been discussed in detail. While damage does not explicitly affect a governing constitutive model, coupling between the fatigue and constitutive model has been treated by introducing a backstress that is an internal variable of the Helmholtz free energy and influences directly both the fatigue damage and the plastic flow.

The capability of the approach under uni- and multi-axial loadings was demonstrated in light of experimental observations for a technologically important PC polymer. Since damage does not affect macroscopic stress reduction, the proposed approach makes it possible to predict the fatigue life of an entire structural element by solely using the single points wherein the damage intensely evolves under the recurrent load spectrum. To demonstrate this, the approach was implemented in a finite-element setting, and the first part of the fatigue experiment of a uniaxial tensile bar was simulated. A microscopic, rectangular region incorporating a packing of inhomogeneities was then exploited as a RVE of the most damaged location. Simulations, in accordance with the model assumption, indicated that damage develops in limited zones around involved inhomogeneities while majority of the material shows considerably less damage and remains virtually homogeneous. The results also showed that fatigue damage development in the polymer matrix occurs at the sites closely following the localization of the plastic deformation and is also influenced by a build-up of hydrostatic stress.

The proposed approach could be developed further. For instance, solely uniaxial, accelerated fatigue experiments are not sufficient to account for directional fatigue damage inherent to amorphous solids when macroscopic cracking initiates $(D=1)$ and results in directional damage fields finally in large strains. Also, the proposed endurance surface needs to be refined so as to govern better the fatigue development under compression. Considering low-cycle fatigue, the impact of temperature rice on energy dissipation increases and should also be included in the modeling. Moreover, several factors, quite ambiguous at the moment, control fatigue via the morphological and microstructural changes and need to be investigated further. Examples of such important supplements are particle size, particle number density, matrix-particle adhesion strength, and disentanglement of a polymer chain network around particles.

\section{Acknowledgements}

The authors acknowledge valuable comments and discussions with Professor Erik van der Giessen. The authors would also like to thank the IT Center for Science Ltd (CSC) for providing resources for the Abaqus software under SIMULIA/Dassault Systemes software licenses.

\section{Appendix A.}


As an example, considering a potential function,

$$
\phi^{\mathrm{d}}=\frac{B_{1} B_{2}}{b+1}\left(\frac{Y}{B_{2}}\right)^{\mathrm{b}+1}
$$

where $Y$ is given by (43) and $B_{1}\left(\mathrm{~s}^{-1}\right), B_{2}$ (MPa), and $b$ are positive parameters, see Ayoub et al. (2011). For the sake of simplicity, $B_{1}=1 \mathrm{~s}^{-1}$ can be chosen.

Substitution of (A.1) into (40) yields

$$
\dot{D}=B_{1}\left(\frac{Y}{B_{2}}\right)^{\mathrm{b}}
$$

and using (43) in (A.2) gives

$$
Y:=-\frac{\partial \varphi^{\mathrm{d}}}{\partial D}=B_{2}\left(\frac{\dot{D}}{B_{1}}\right)^{\frac{1}{\mathrm{~b}}} \geq 0
$$

which result is thermodynamically consistent for all positive $Y$ and for all decreasing functions $\varphi^{\mathrm{d}}$ (damage never decreases), see Theorem 2.3 .

What follows is that one is free to select the expression (56), i.e.

$$
\dot{D}=K \exp \left(f\left(\beta ; L_{1}, L_{2}, \vartheta\right)\right) \dot{\beta} \geq 0
$$

for damage evolution in the dissipation inequality (50).

\section{References}

Abaqus, 2012. Abaqus Analysis User's Manual, Version 6.12. Technical Report. Dassault Systémes Simulia Corp., Providence, RI, USA.

Agah-Tehrani, A., Lee, E.H., Mallett, R.L., Onat, E.T., 1987. The theory of elastic-plastic deformation at finite strain with induced anisotropy modeled as combined isotropic-kinematic hardening. J. Mech. Phys. Solids 35, 519-539.

Anand, L., Ames, N.M., 2006. On modeling the micro-indentation response of an amorphous polymer. Int. J. Plasticity $22,1123-1170$.

Anand, L., Gearing, B.P., 2004. On modeling the deformation and fracture response of glassy polymers due shear-yielding and crazing. Int. J. Solids Structures 41, 3125-3150.

Anand, L., Gurtin, M.E., 2003. A theory of amorphous solids undergoing large deformations with application to polymer glasses. Int. J. Solids Structures 40, 1465-1487.

Arruda, E.M., Boyce, M.C., Quintus-Bosz, H., 1993. Effects of initial anisotropy on the finite strain deformation behavior of glassy polymers. Int. J. Plasticity 9, 783-811.

Ayoub, G., Naït-Abdelaziz, M., Zaïri, F., Gloaguen, J.M., Charrier, P., 2011. A continuum damage model for the high-cycle fatigue life prediction of styrene-butadiene rubber under multiaxial loading. Int. J. Solids Structures 48, 2458-66.

Bannantine, J.A., Comer, J.J., Handrock, J.L., 1990. Fundamentals of Metal Fatigue Analysis (1st edition). Prentice Hall. 
Beesley, R., Chen, H., Hughes, M., 2017. A novel simulation for the design of a low cycle fatigue experimental testing programme. Comp. Struct. 178, 105-118.

Bergström, J.S., Boyce, M.C., 1998. Constitutive modeling of the large strain time-dependent behavior of elastomers. J. Mech. Phys. Solids 46, 931-954.

Boyce, M.C., Parks, D.M., Argon, A.S., 1988. Large inelastic deformation of glassy polymers, Part I: Rate-dependent constitutive model. Mechanics of Materials 7, 15-33.

Boyce, M.C., Weber, G.G., Parks, D.M., 1989. On the kinematics of finite strain plasticity. J. Mech. Phys. Solids 37, 647-665.

Chaboche, J.L., 1997. Thermodynamic formulation of constitutive equations and application to the viscoplasticity of metals and polymers. Int. J. Solids Structures 34, 2239-2254.

Dal, H., Kaliske, M., 2009. Bergström-Boyce model for nonlinear finite rubber viscoelasticity: theoretical aspects and algorithmic treatment for the FE method. Comp. Mech. 44, 809 823 .

Ding, G., Karlsson, A.M., Santare, M.H., 2017. Numerical evaluation of fatigue crack growth in polymers based on plastically dissipated energy. Int. J. Fatigue 94, 89-96.

Dreistadt, C., Bonnet, A.S., Chevrier, P., Lipinski, P., 2009. Experimental study of the polycarbonate behaviour during complex loadings and comparison with the Boyce, Parks and Argon model predictions. Materials and Design 30, 3126-3140.

Dündar, H., Ayhan, A.O., 2015. Three-dimensional fracture and fatigue crack propagation analysis in structures with multiple cracks. Comp. Struct. 158, 259-273.

Engqvist, J., Wallin, M., Ristinmaa, M., Hall, S.A., Plivelic, T.S., 2016. Modelling multi-scale deformation of amorphous glassy polymers with experimentally motivated evolution of the microstructure. J. Mech. Phys. Solids 96, 497-510.

Fatemi, A., Yang, L., 1998. Cumulative fatigue damage and life prediction theories: a survey of the state of the art for homogeneous materials. Int. J. Fatigue 20, 9-34.

Foster, A.M., 2015. Materials Testing Standards for Additive Manufacturing of Polymer Materials: State of the Art and Standards Applicability. Technical Report. Materials and Structural Systems Division Engineering Laboratory, NISTIR 8059

Haward, R.N., Thackray, G., 1968. The use of a mathematical model to describe isothermal stress-strain curves in glassy thermoplastics. Proc. Roy. Soc. A. 302, 453-472.

Hoger, A., 1986. The material time derivative of logarithmic strain. Int. J. Solids Structures $22,1019-32$

Holopainen, S., 2013. Modeling of the mechanical behavior of amorphous glassy polymers under variable loadings and comparison with state-of-the-art model predictions. Mechanics of Materials 66, 35-58.

Holopainen, S., 2014. Representations of $m$-linear functions on tensor spaces. Duals and transpositions with applications in continuum mechanics. Mathematics and Mechanics of Solids 19, 168-193.

Holopainen, S., Barriere, T., Cheng, G., Kouhia, R., 2017. Continuum approach for modeling fatigue in amorphous glassy polymers. applications to the investigation of damageratcheting interaction in polycarbonate. Int. J. Plasticity 91, 109-133.

Holopainen, S., Kouhia, R., Saksala, T., 2016. Continuum approach for modeling transversely isotropic high-cycle fatigue. European Journal of Mechanics A/Solids 60, 183-195. 
Hughes, J.M., Lugo, M., Bouvard, J.L., McIntyre, T., Horstemeyer, M.F., 2017. Cyclic behavior and modeling of small fatigue cracks of a polycarbonate polymer. Int. J. Fatigue $99,78-86$.

Janssen, R.P.M., Kanter, D.K., Govaert, L.E., Meijer, H.E.H., 2008. Fatigue life predictions for glassy polymers: A constitutive approach. Macromolecules 41, 2520-30.

Kanters, M.J.W., Kurokawa, T., Govaert, L.E., 2016. Competition between plasticitycontrolled and crack-growth controlled failure in static and cyclic fatigue of thermoplastic polymer systems. Polymer Testing 50, 101-110.

Khan, F., Lopez-Pamies, O., Kazmi, R., 2006. Thermo-mechanical large deformation response and constitutive modeling of viscoelastic polymers over a wide range of strain rates and temperatures. Int. J. Plasticity 22, 581-601.

Kim, G.H., Lu, H., 2008. Accelerated fatigue life testing of polycarbonate at low frequency under isothermal condition. Polymer Testing 27, 114-121.

Kwang, S.C., 2016. Viscoelasticity of Polymers: Theory and Numerical Algorithms. Springer.

Launeya, M.E., Hofmann, D.C., Johnson, W.L., Ritchie, R.O., 2009. Solution to the problem of the poor cyclic fatigue resistance of bulk metallic glasses. Proceedings of the National Academy of Sciences of the United States of America (PNAS) 106, 4986-91.

Lawrimore, W.B., Francis, D.K., Bouvard, J.L., Hammi, Y., Horstemeyer, M.F., 2016. A mesomechanics parametric finite element study of damage growth and coalescence in polymers using an Elastoviscoelastic-Viscoplastic internal state variable model. Mechanics of Materials 96, 83-95.

Lemaitre, J., Desmorat, R., 2005. Engineering Damage Mechanics. Ductile, Creep, Fatigue and Brittle Failures. Springer-Verlag, Berlin.

Lesser, A.J., 2002. Fatigue behavior of polymers. In: Encyclopedia of Polymer Science and Technology 6, 197-251.

Li, X., Hristov, H.A., Yee, A.F., Gidley, D.W., 1995. Influence of cyclic fatigue on the mechanical properties of amorphous polycarbonate. Polymer 36, 759-765.

Lu, F., Kang, G., Zhu, Y., Xi, C., Jiang, H., 2016. Experimental observation on multiaxial ratchetting of polycarbonate polymer at room temperature. Polymer Testing 50, 135-144.

Lubliner, J., 1985. A model of rubber viscoelasticity. Mech Res Commun 12, 93-99.

Lugo, M., Fountain, J.E., Hughes, J.M., Bouvard, J.L., Horstemayer, M.F., 2014. Microstructure-based fatigue modeling of an acrylonitrile butadiene styrene (abs) copolymer. Journal of Applied Polymer Science 131, 1-12.

Marissen, R., Schudy, D., Kemp, A.V.J.M., Coolen, S.M.H., Duijzings, W.G., der Pol, A.V., Gulick, A.J.V., 2001. The effect of material defects on the fatigue behaviour and the fracture strain of ABS. Journal of Material Sciences 36, 4167-80.

Maxwell, A.S., Broughton, W.R., Dean, G., Sims, G.D., 2005. Review of accelerated ageing methods and lifetime prediction techniques for polymeric materials. Technical Report. National Physical Laboratory Hampton Road, Teddington, Middlesex, TW11 0LW.

Melick, H.G.H.V., 2003. Deformation and failure of polymer glasses. Doctoral thesis. University Press Facilities, Eindhoven.

Miehe, C., Stein, E., Wagner, W., 1993. Associative multiplicative elasto-plasticity formulation and aspects of the numerical implementation including stability analysis. Comp. Struct. 52, 969-978. 
Murakami, S., 2012. Continuum Damage Mechanics: A Continuum Mechanics Approach to the Analysis of Damage and Fracture. Springer Science and Business Media.

Ottosen, N., Stenström, R., Ristinmaa, M., 2008. Continuum approach to high-cycle fatigue modeling. Int. J. Fatigue 30, 996-1006.

Reese, S., Govinjee, S., 1998. A theory of finite viscoelasticity and numerical aspects. Int. J. Solids Structures 35, 3455-82.

Ritchie, R.O., 1999. Mechanisms of fatigue-crack propagation in ductile and brittle solids. Int. J. Fracture 100, 55-83.

Sidoroff, F., 1974. Nonlinear viscoelastic model with an intermediate configuration. J Mécaniques 13, 679-713.

Steenbrink, A.C., Van der Giessen, E., 1998. Studies on the growth of voids in amorphous glassy polymers. Journal of Material Sciences 33, 3163-3175.

Stein, E., Sagar, G., 2008. Convergence behavior of 3D finite elements for Neo-Hookean material. Engineering Computations: International Journal for Computer-Aided Engineering and Software 25, 220-232.

Steinmann, P., Stein, E., 1996. On the numerical treatment and analysis of finite deformation ductile single crystal plasticity. Comput. Methods Appl. Mech. Engng. 129, 235-254.

Sun, B.A., Wang, W.H., 2015. The fracture of bulk metallic glasses. Progress in Materials Science 74, 211-307.

Wallin, M., Holopainen, S., 2012. Modeling of long-term behavior of amorphous glassy polymers. ASME J. Engng. Materials Technol. 135, 1-11.

Wang, J., Xu, Y., Zhang, W., Moumni, Z., 2016. A damage-based elastic-viscoplastic constitutive model for amorphous glassy polycarbonate polymers. Materials and Design 97, $519-531$.

Weber, G., Anand, L., 1990. Finite deformation constitutuve equations and a time integration procedure for isotropic, hyperelastic-viscoplastic solids. Comput. Methods Appl. Mech. Engng. 79, 173-202.

Zaïri, F., Naït-Abdelaziz, M., Gloaguen, J.M., Lefebvre, J.M., 2011. A physically-based constitutive model for anisotropic damage in rubber-toughened glassy polymers during finite deformation. Int. J. Plasticity 27, 25-51.

Zaïri, F., Woznica, K., Naït-Abdelaziz, M., 2005. Phenomenological nonlinear modelling of glassy polymers. Comptes Rendus Mecanique 333, 359-364. 